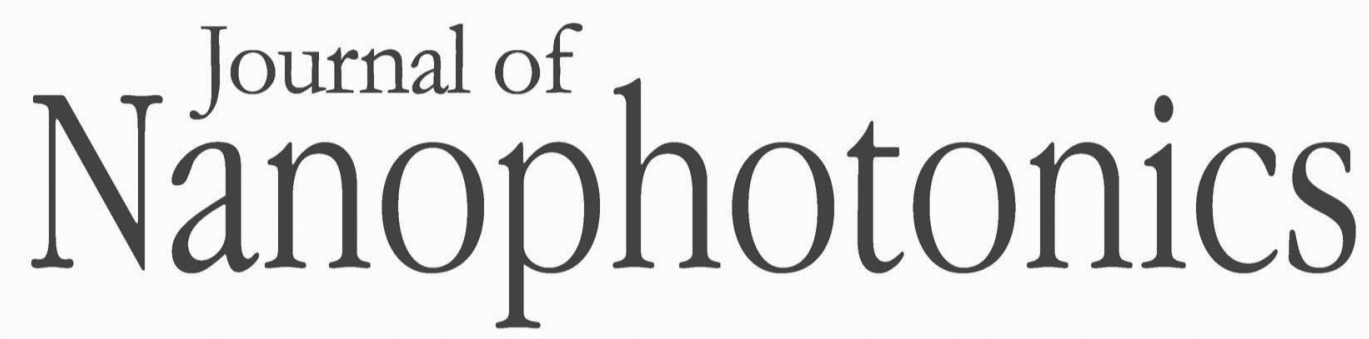

SPIEDigitalLibrary.org/jnp

\title{
Review of recent progress of III-nitride nanowire lasers
}

Shamsul Arafin

Xianhe Liu

Zetian Mi

\section{O SPIE}




\title{
Review of recent progress of III-nitride nanowire lasers
}

\author{
Shamsul Arafin, Xianhe Liu, and Zetian Mi \\ McGill University, Department of Electrical and Computer Engineering, \\ 3480 University Street, Montreal, Quebec H3A 0E9, Canada \\ shamsul.arafin@mcgill.ca
}

\begin{abstract}
One-dimensional compound semiconductor nanolasers, especially nanowire (NW)based nanolasers utilizing III-nitride (AlGaInN) materials system, are an emerging and promising area of research. Significant achievements have been made in developing III-nitride NW lasers with emission wavelengths from the deep ultraviolet (UV) to the near-infrared spectral range. The types of lasers under investigation include Fabry-Pérot, photonic crystal, plasmonic, ring resonator, microstadium, random, polariton, and two-dimensional distributed feedback lasers. The lasing thresholds vary by several orders of magnitude, which are a direct consequence of differing NW dimensions, quality of the NWs, characteristics of NW cavities, and coupling with the substrate. For electrically injected, such as ultralow-threshold and continuous-wave III-nitride NW lasers that can operate at room temperature, the following obstacles remain: carrier loss mechanisms including defect-related nonradiative surface recombination, electron overflow, and poor hole transport; low radiative recombination efficiency and high surface recombination; poor thermal management; and highly resistive ohmic contacts on the $p$-layer. These obstacles must be overcome to fully realize the potential of these lasers. (c) 2013 Society of Photo-Optical Instrumentation Engineers (SPIE) [DOI: 10.1117/1.JNP.7.074599]
\end{abstract}

Keywords: III-nitride nanowires; nanowire heterostructures; nanolasers; GaN; photoluminescence; Fabry-Pérot; plasmonics; random lasing.

Paper 13045V received Jun. 2, 2013; revised manuscript received Aug. 7, 2013; accepted for publication Aug. 9, 2013; published online Sep. 20, 2013.

\section{Introduction}

Semiconductor one-dimensional (1-D) nanostructures have emerged as a key component for a wide range of electronic and optoelectronic applications since their first demonstration in $1964{ }^{1,2}$ Currently, a great deal of attention has been paid to semiconductor nanowires (NWs) due to their unique electronic and optical properties arising from the anisotropic geometry, the large surface-to-volume ratios, and the unique carrier and photon confinement in two dimensions. One of the most attractive advantages of NWs is that they can be grown either with or without an epitaxial connection to a substrate, which relieves us from lattice and thermal expansion coefficients mismatch problems in these structures. ${ }^{3-5}$ This results in, in principle, materials that are free from strain, dislocations, and other structural defects. Therefore, NWs hold great promise in a number of applications including electronics, ${ }^{6,7}$ photovoltaics, ${ }^{8}$ solidstate lighting, ${ }^{9}$ and biosensing. ${ }^{10}$

Furthermore, in order to increase the applicability of NWs as light sources, recent investigations have been focused on making semiconductor lasers utilizing NWs compatible with very large scale integration (VLSI) circuits. In other words, implementing such digital photonic VLSI systems requires lasers at nanoscale dimensions. Apart from that, lasers with a reduced dimensionality will also allow for developing efficient, low-threshold, and compact light sources for many novel applications in high-speed communications, information processing, and optical interconnects. Thus, there is an urgent need to reduce the size of semiconductor lasers. As a consequence, size reduction of lasers has become one of the most interesting and challenging areas in modern optoelectronics. This is not only for using them as light sources in such

0091-3286/2013/\$25.00 두 2013 SPIE 
applications, but also for understanding the basic laser concepts and fundamental light-matter interactions at the nanoscale.

The smallest application-suited lasers available commercially today are vertical-cavity surface-emitting lasers (VCSELs). ${ }^{11}$ Compared with VCSELs, NW lasers can exhibit much smaller dimensions with diameters of $\sim 100 \mathrm{~nm}$ or less, and therefore, they promise much lower power consumption and higher integration density. It should be noticed that in spite of their small cylindrical dimensions, their lengths can vary from a few hundred nanometers to several or tens of micrometers. Also, NWs as gain regions are expected to exhibit superior performance due to their strong carrier confinement and enhanced electronic density of states. In addition, nearly defect-free NWs can be grown directly on silicon (Si) substrates, ${ }^{12-17}$ thereby allowing for the monolithic integration of efficient light emitters with other electronic and photonic components on a Si platform for the emerging chip-level optical communications.

NW lasers utilizing II-VI compounds, e.g., $\mathrm{ZnO}^{18-24}$ and $\mathrm{CdS},{ }^{25-27} \mathrm{III}-\mathrm{V}$ compounds, e.g., GaAs and GaSb, ${ }^{28,29}$ and their ternary alloys, e.g., CdSSe and InGaAs, ${ }^{30,31}$ have been demonstrated. Recently, III-nitride NW-based lasers ${ }^{32-48}$ have attracted a great deal of interest. The direct bandgap III-nitrides ( $\mathrm{AlN}, \mathrm{GaN}$, and $\mathrm{InN}$ ) and their ternary and quaternary alloys exhibit many unique properties including relatively large oscillator strength, ${ }^{49,50}$ large exciton binding energy, ${ }^{51}$ low surface state densities, ${ }^{52}$ large band offset, and robust spin coherence. ${ }^{53}$ In particular, owing to the large exciton binding energy ( $26 \mathrm{meV}$ at room temperature), GaN materials can have excitonic effects up to room temperature, which could be exploited to realize polariton lasers. ${ }^{54}$ More importantly, III-nitride NW lasers can potentially cover a broad spectral range from the deep $\mathrm{UV}(6.2 \mathrm{eV}$ for $\mathrm{AlN})$ to the near-infrared $(0.7 \mathrm{eV}$ for $\mathrm{InN})$ regime, as illustrated in Fig. 1. Therefore, III-nitride NW lasers grown on Si substrate will also enable many potential applications in optical information processing and quantum computing.

Given the rapid developments in III-nitride NW lasers, there is clearly an urgent need for a comprehensive review of the recent progress and research activities. Compared with previously published review papers on NW lasers, ${ }^{55-61}$ this review paper will provide a detailed description of III-nitride NW lasers. The recent progresses and challenges for such nanoscale lasers are first briefly summarized in Secs. 2 and 3, respectively. The operating principles of NW lasers are then described in Sec. 4. Several growth techniques of NWs are briefly mentioned in Sec. 5. This will be followed by discussions on the recent demonstrations of various III-nitride NW lasers in Sec. 6. Finally, a summary on the future prospects of III-nitride NW lasers is presented in Sec. 7.

\section{Overview of the Recent Progress of III-Nitride Nanowire Lasers}

NWs synthesized from III-nitride semiconductors have recently shown tremendous promise for different types of nanophotonic devices including lasers, light-emitting diodes (LEDs), ${ }^{62-66}$ and photodetectors. ${ }^{67-70}$ As solid-state light sources, III-nitride NW-based devices provide many potential advantages when compared with their planar counterparts. Conventional planar III-nitride lasers grown on sapphire and $\mathrm{SiC}$ substrates exhibit very large dislocation densities. ${ }^{71-73}$ Moreover, the performance of such laser diodes has been severely limited by the large lattice mismatch between $\mathrm{InGaN}$ and $\mathrm{GaN}$ and the associated strain-induced polarization fields. $^{74,75}$ This leads to extremely large threshold current densities of $\sim 10 \mathrm{kA} / \mathrm{cm}^{2}$ or larger for

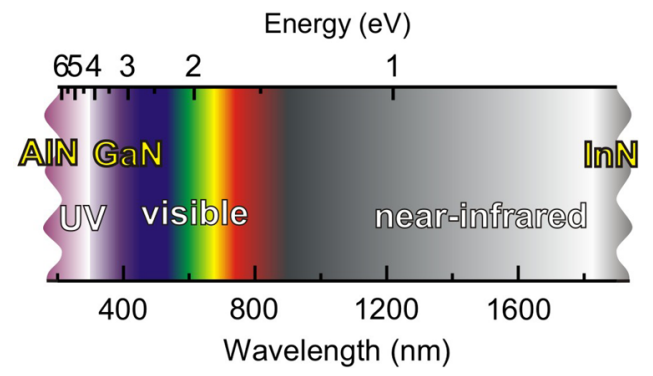

Fig. 1 Optical spectrum showing the coverage of the wide wavelength range from the deep ultraviolet (UV) to the near-infrared range by the III-nitride material system. 
devices emitting in the wavelength $(\lambda)$ range beyond $\sim 500 \mathrm{~nm}^{76-80}$ In contrast, NW-based devices can exhibit drastically reduced dislocation densities and polarization fields and can provide a greater degree of flexibility for sophisticated device engineering. ${ }^{66,81}$ Therefore, III-nitride NW lasers are expected to exhibit much reduced threshold current densities and lower power consumption. Moreover, such nanoscale lasers with a reduced cavity volume can potentially operate at much higher modulation speed. ${ }^{82}$ In addition to these significant advantages, NW-based lasers can promise droop-free operation. ${ }^{83}$

With the use of III-nitride NW structures, nanolasers with emission wavelengths ranging from the deep UV to the near-infrared have already been demonstrated. Many different types of micro- and nanocavities have been studied which include Fabry-Pérot ${ }^{32-34,36,38,46-48}$ nanoplasmonics, ${ }^{43,45}$ photonic-crystals, ${ }^{40}$ microstadium, ${ }^{37}$ and ring cavities. ${ }^{35}$ GaN-based NW polariton laser also has been demonstrated. ${ }^{42}$ Stimulated emission has been reported utilizing two-dimensional (2-D) distributed feedback (DFB) concept in an InGaN nanocolumn array ${ }^{39,41}$ In addition, random lasing action from GaN quasicrystal nanorod arrays ${ }^{44}$ has been observed. Recently, stable single-mode lasing operation from a single ${ }^{46,48}$ or a pair ${ }^{47}$ of coupled GaN NWs was demonstrated. Table 1 provides a brief summary of III-nitride nanolasers and their corresponding performance parameters demonstrated by different research teams in the world. These NW laser demonstrations generally involve the use of optical pumping, and the achievement of electrically injected III-nitride NW lasers has remained elusive.

It may be noticed that the lasing thresholds of these lasers vary by several orders of magnitude, which is a direct consequence of differing NW dimensions, quality of the NWs, characteristics of NW cavities, and coupling with the substrate.

\section{Challenges of III-Nitride Nanowire Lasers}

For practical applications, it is highly desired to realize electrically injected, ultralow-threshold, and continuous-wave (CW) III-nitride NW lasers that can be operated at room temperature. Some of the major obstacles and difficulties for realizing such devices are briefly described below:

- Due to the highly effective lateral stress relaxation associated with the large surface areas, both the polarization fields and the dislocation densities in NW devices can be significantly reduced. However, the performance of III-nitride NW devices still suffers from several carrier loss mechanisms including defect-related nonradiative surface recombination, ${ }^{84}$ electron overflow, ${ }^{85,86}$ and poor hole transport. ${ }^{87}$ Additionally, it is still under debate whether Auger recombination plays an important role in the performance of nitridebased devices. ${ }^{88,89}$

- The performance of NW-based devices may be further degraded by the presence of radialband bending and surface Fermi-level pinning. Injected charge carriers, i.e., electrons and holes, may be spatially separated, which leads to a reduced radiative recombination efficiency and an enhanced surface recombination. ${ }^{90,91}$

- The performance of semiconductor lasers, including the output power and threshold current, is often limited by thermal effects. For NWs with diameters smaller than the bulk mean free path of hot carriers, theory predicts that the thermal conductivity will be much reduced when compared with their bulk counterparts. The thermal conductivity of single GaN NWs with different diameters has been reported. ${ }^{92-94}$ Unexpectedly small values, in the range of 13 to $19 \mathrm{~W} / \mathrm{m}-\mathrm{K}$ at $300 \mathrm{~K}$, have been observed. ${ }^{92}$ This clearly indicates the critical importance of the thermal management of NW lasers.

- To date, the controlled incorporation of $n$ - and $p$-type dopants in NW structures have remained challenging, which makes it difficult to achieve electrically injected devices. Recent studies have shown that Si-dopants are preferentially incorporated in the nearsurface region of III-nitride NWs, due to the smaller formation energy for substitutional Si-doping at the surface. ${ }^{95,96} \mathrm{In}$ addition, the achievement of $p$-type doping in $\mathrm{InGaN}$ and InAlN with high In and $\mathrm{Al}$ compositions has still been a big concern. As a consequence, it is difficult to make good quality and low-resistive ohmic contacts on the $p$-layer of this material system. 
Table 1 A brief summary of experimental realizations of III-nitride NW lasers listed in chronological order.

\begin{tabular}{|c|c|c|c|c|c|c|}
\hline Research team (year) & $\begin{array}{l}\text { Active } \\
\text { layer }\end{array}$ & Substrate & $\begin{array}{l}\text { Max. } \\
\text { operating } \\
\text { temp }\end{array}$ & Threshold & $\lambda(\mathrm{nm})$ & $\begin{array}{l}\text { Resonator } \\
\text { type }\end{array}$ \\
\hline $\begin{array}{l}\text { Univ. of California, Berkeley } \\
\text { and Lawrence Berkeley } \\
\text { National Laboratory, Berkeley, } \\
\text { CA (2002) }\end{array}$ & GaN & c-Sapphire & $\mathrm{RT}$ & $700 \mathrm{~nJ} / \mathrm{cm}^{2}$ & $\begin{array}{l}370 \text { to } \\
380\end{array}$ & Fabry-Pérot \\
\hline $\begin{array}{l}\text { Univ. of California, Berkeley } \\
\text { and Lawrence Berkeley } \\
\text { National Laboratory, Berkeley, } \\
\text { CA (2003) }\end{array}$ & GaN & c-Sapphire & RT & $2-3 \mu \mathrm{J} / \mathrm{cm}^{2}$ & 384 & Fabry-Pérot \\
\hline $\begin{array}{l}\text { Harvard Univ., } \\
\text { Cambridge, MA (2005) }\end{array}$ & $\mathrm{GaN}$ & c-Sapphire & $\mathrm{RT}$ & $22 \mathrm{~kW} / \mathrm{cm}^{2}$ & $\begin{array}{l}370 \text { to } \\
378\end{array}$ & Fabry-Pérot \\
\hline $\begin{array}{l}\text { Univ. of California, Berkeley } \\
\text { and Lawrence Berkeley } \\
\text { National Laboratory, Berkeley, } \\
\text { CA (2006) }\end{array}$ & GaN & c-Sapphire & RT & $112.5 \mu \mathrm{J} / \mathrm{cm}^{2}$ & $\begin{array}{c}373 \text { to } \\
380\end{array}$ & Ring \\
\hline $\begin{array}{l}\text { Academia Sinica, Taipei, } \\
\text { National Taiwan Univ., Taipei, } \\
\text { National Taiwan Ocean Univ., } \\
\text { Keelung and National Taiwan } \\
\text { Univ. of Science and Tech., } \\
\text { Taipei (2007) }\end{array}$ & $\ln N$ & Silicon & $20 \mathrm{~K}$ & $70 \mathrm{~kW} / \mathrm{cm}^{2}$ & $\begin{array}{l}1559 \text { to } \\
1644\end{array}$ & Fabry-Pérot \\
\hline $\begin{array}{l}\text { Korea Univ., Seoul and } \\
\text { Harvard Univ., Cambridge, MA } \\
\text { (2007) }\end{array}$ & GaN & c-Sapphire & $\mathrm{RT}$ & $1536 \mathrm{~kW} / \mathrm{cm}^{2}$ & $\begin{array}{l}370 \text { to } \\
372\end{array}$ & Microstadium \\
\hline $\begin{array}{l}\text { Harvard Univ., Cambridge, MA, } \\
\text { Georgia Institute of Tech, } \\
\text { Atlanta, GA, Univ. of California, } \\
\text { Santa Cruz, CA, } \\
\text { Massachusetts Institute of } \\
\text { Tech., Cambridge, MA, and } \\
\text { Korea Univ. Seoul (2008) }\end{array}$ & $\begin{array}{l}\text { InGaN/ } \\
\text { GaN }\end{array}$ & r-Sapphire & $\mathrm{RT}$ & $900 \mathrm{~kW} / \mathrm{cm}^{2}$ & $\begin{array}{l}383 \text { to } \\
478\end{array}$ & Fabry-Pérot \\
\hline $\begin{array}{l}\text { Sophia Univ. and Japan } \\
\text { Science and Technology } \\
\text { Agency, Tokyo (2009) }\end{array}$ & $\begin{array}{l}\text { InGaN/ } \\
\text { GaN }\end{array}$ & $\begin{array}{l}\text { GaN/c- } \\
\text { Sapphire } \\
\text { template }\end{array}$ & $\mathrm{RT}$ & $320 \mathrm{~kW} / \mathrm{cm}^{2}$ & 470 & 2-D DFB \\
\hline $\begin{array}{l}\text { University of Michigan, Ann } \\
\text { Arbor, Ml (2011) }\end{array}$ & $\mathrm{GaN}$ & Silicon & $\mathrm{RT}$ & $120 \mathrm{~kW} / \mathrm{cm}^{2}$ & 370 & $\begin{array}{l}\text { Photonic } \\
\text { crystal }\end{array}$ \\
\hline $\begin{array}{l}\text { Sophia Univ. and Japan } \\
\text { Science and Tech. Agency, } \\
\text { Tokyo (2011) }\end{array}$ & $\begin{array}{l}\text { InGaN/ } \\
\text { GaN }\end{array}$ & $\begin{array}{l}\text { GaN/c- } \\
\text { sapphire } \\
\text { template }\end{array}$ & $\mathrm{RT}$ & $0.42 \mathrm{MW} / \mathrm{cm}^{2}$ & $\begin{array}{l}440 \text { to } \\
560\end{array}$ & 2-D DFB \\
\hline $\begin{array}{l}\text { University of Michigan, Ann } \\
\text { Arbor, Ml (2011) }\end{array}$ & $\mathrm{GaN}$ & Silicon & $\mathrm{RT}$ & $92.5 \mathrm{~nJ} / \mathrm{cm}^{2}$ & $\begin{array}{l}366 \text { to } \\
372\end{array}$ & Polariton \\
\hline $\begin{array}{l}\text { National Tsing-Hua Univ. and } \\
\text { National Chiao-Tung Univ., } \\
\text { Hsinchu (2011) }\end{array}$ & $\begin{array}{l}\text { InGaN/ } \\
\text { GaN }\end{array}$ & Silicon & $7 \mathrm{~K}$ & $300 \mathrm{~kW} / \mathrm{cm}^{2}$ & 533 & Plasmonic \\
\hline $\begin{array}{l}\text { National Chiao Tung Univ., } \\
\text { Hsinchu, Academia Sinica, } \\
\text { Taipei, Luxtaltek corporation, } \\
\text { Miaoli and National Chiao Tung } \\
\text { Univ., Hsinchu (2012) }\end{array}$ & GaN & $\begin{array}{l}\text { GaN/c- } \\
\text { sapphire } \\
\text { template }\end{array}$ & $\mathrm{RT}$ & $5 \mathrm{MW} / \mathrm{cm}^{2}$ & $\begin{array}{l}455 \text { to } \\
470\end{array}$ & Random \\
\hline $\begin{array}{l}\text { National Tsing-Hua Univ., } \\
\text { Hsinchu, Univ. Texas, Austin, } \\
\text { TX, National Chiao Tung Univ., } \\
\text { Hsinchu, Chinese Academy of } \\
\text { Sciences, Beijing (2012) }\end{array}$ & $\begin{array}{c}\text { InGaN/ } \\
\text { GaN }\end{array}$ & Silicon & $78 \mathrm{~K}$ & $3.7 \mathrm{~kW} / \mathrm{cm}^{2}$ & $\begin{array}{l}510 \text { to } \\
522\end{array}$ & Plasmonic \\
\hline
\end{tabular}


Table 1 (Continued).

\begin{tabular}{|c|c|c|c|c|c|c|}
\hline Research team (year) & $\begin{array}{l}\text { Active } \\
\text { layer }\end{array}$ & Substrate & $\begin{array}{l}\text { Max. } \\
\text { operating } \\
\text { temp }\end{array}$ & Threshold & $\lambda(\mathrm{nm})$ & $\begin{array}{l}\text { Resonator } \\
\text { type }\end{array}$ \\
\hline $\begin{array}{l}\text { Sandia National Laboratories } \\
\text { and Univ. New Mexico, } \\
\text { Albuquerque, NM (2012) }\end{array}$ & GaN & c-Sapphire & $\mathrm{RT}$ & $231 \mathrm{~kW} / \mathrm{cm}^{2}$ & $\begin{array}{c}367 \text { to } \\
371\end{array}$ & Fabry-Pérot \\
\hline $\begin{array}{l}\text { Univ. New Mexico and Sandia } \\
\text { National Laboratories, } \\
\text { Albuquerque, NM (2012) }\end{array}$ & GaN & c-Sapphire & $\mathrm{RT}$ & $874 \mathrm{~kW} / \mathrm{cm}^{2}$ & $\begin{array}{c}370 \text { to } \\
374\end{array}$ & Fabry-Pérot \\
\hline $\begin{array}{l}\text { Univ. New Mexico, Sandia } \\
\text { National Laboratories, } \\
\text { Albuquerque, NM and } \\
\text { University of Essex, } \\
\text { Colchester (2012) }\end{array}$ & GaN & c-Sapphire & $\mathrm{RT}$ & $241 \mathrm{~kW} / \mathrm{cm}^{2}$ & 370 & Fabry-Pérot \\
\hline
\end{tabular}

- A detailed understanding and precise control of the NW structural, optical and electrical transport properties are of utmost requirements to design high-performance NW nanolasers. The lack of detailed knowledge on these properties represents another limiting factor for further improving the performance of nitride NW-based lasers.

\section{Operating Principle of Nanowire Lasers}

The realization of NW lasers opens up a new and plausible route for achieving simultaneous carrier and photon confinements in 1-D nanoscale cavities. Compared with quantum-well structures, a better carrier confinement in 1-D structures allows carriers to move freely only along the wire axial direction. Optical confinement in miniature optical cavities can lead to a significant enhancement of the spontaneous emission rate. ${ }^{97}$ Note that spontaneous emission is no longer an intrinsic material property, but rather it is directly proportional to the density of electromagnetic modes with which a spontaneously emitted photon can couple. This cavity quantum electrodynamics phenomenon can provide a mechanism to realize low-threshold semiconductor lasers from NW-based cavities.

A number of different optical resonators ranging from Fabry-Pérot, ${ }^{32-34,36,38,46,47}$ plasmonics, ${ }^{43,45}$ microstadiums,${ }^{37}$ photonics crystals, ${ }^{40}$ 2-D-DFB, ${ }^{39,41}$ ring, ${ }^{35}$ and random cavities have been utilized for the realization of III-nitride nanolasers. Each resonator has its own unique properties based on which lasing occurs in the device. Regardless of the types of the resonator, generally speaking, all nanolasers should have a gain medium for compensating all types of cavity losses, as well as a feedback mechanism to provide sustained laser oscillation. This section will mainly focus on the fundamental operating principles of nanolasers using the simplest Fabry-Pérot (FP) cavity. This will be followed by discussions on the unique features of NW lasers with respect to standard semiconductor lasers. And last, but not least, a short description about the basic operation of plasmonic NW lasers will be provided.

Perhaps the smallest FP-type optical cavity that can effectively provide positive feedback of stimulated emission belongs to single-crystalline semiconductor NWs having a length on the order of light wavelength. Figure 2(a) shows a possible geometry of single-NW lasers representing FP nanocavity that can be fabricated from semiconductor 1-D nanostructures. Two crystalline NW facet ends acting as reflecting mirrors are used in such nanocavities. As the NW cavity is identical to a gain medium terminated by two reflectors, the threshold conditions for the NW can then be described by the balance between the round-trip gain and loss inside the cavity. Optical field propagating along the longitudinal direction is amplified and absorbed inside the NWs. Furthermore, part of the light is reflected back into the cavity from the facets of the NW, and the remaining light emits from the facets to the surrounding medium, as illustrated in Fig. 2(b). Hence, the threshold condition for laser oscillation is that the round-trip gain inside the NW cavity equals the round-trip losses. 


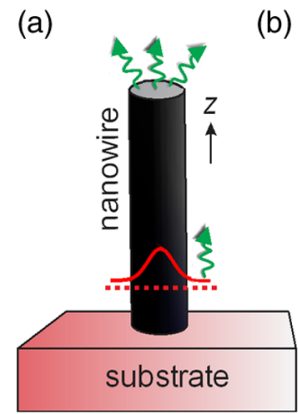

(b)
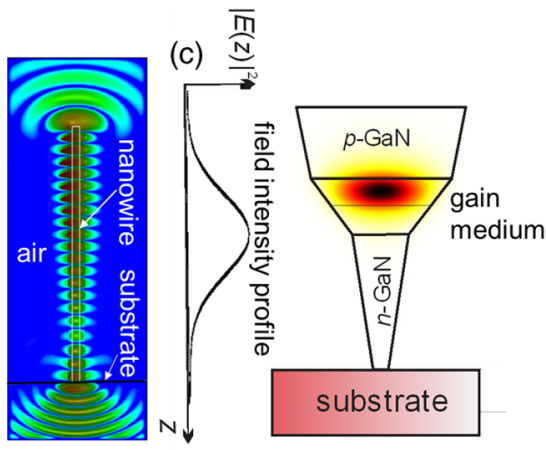

Fig. 2 (a) Schematic of a single NW Fabry-Pérot (FP) cavity. (b) Laser intensity profile along a nanowire (NW) laser. The NW laser is confined at the bottom by the substrate and surrounded by air from all other sides. Reproduced with permission from Ref. 98. (c) The gain medium inside a NW with tapered geometry is sandwiched between the top and the bottom cladding layers, showing the good optical confinement.

$$
\begin{gathered}
\Gamma_{0} g_{\mathrm{th}}=\alpha_{\mathrm{w}}+\frac{1}{2 L} \ln \frac{1}{R_{\mathrm{f}} R_{\mathrm{r}}} \\
\alpha_{\mathrm{m}}=\frac{1}{2 L} \ln \frac{1}{R_{\mathrm{f}} R_{\mathrm{r}}},
\end{gathered}
$$

where $\Gamma_{0}$ is the confinement factor, $g_{\mathrm{th}}$ is the threshold gain, $\alpha_{\mathrm{w}}$ is the waveguide loss, $\alpha_{\mathrm{m}}$ is the mirror loss, $L$ is the length of the NW, and $R_{\mathrm{f}}$ and $R_{\mathrm{r}}$ are the intensity reflectivities of the two end facets of the NW. It should be noted that the gain length in such nanoscale lasers is not necessarily equal to the length of an NW even if it is under optical pumping, since the whole NW is not generally pumped. The similar condition also holds for electrically pumped structures. Therefore, the threshold condition for NW lasers follows

$$
2 \Gamma_{0} g_{\mathrm{th}} L_{\mathrm{g}}=2 \alpha_{\mathrm{w}} L+\ln \frac{1}{R_{\mathrm{f}} R_{\mathrm{r}}},
$$

where $L_{\mathrm{g}}$ is the gain length within the NW.

In contrast to conventional edge-emitting lasers, the dominant loss mechanism is considered to be the mirror loss in NW lasers, i.e., transmission at end facets, due to the much smaller cavity length and reflection coefficient. In addition, significant scattering losses appear in such nanoscale devices when the diameter of the NW is comparable with or smaller than the wavelength of light in the semiconductor. This results in a considerable extension of optical field outside the NW. This adds up to the mirror losses, because only the fraction of the field that is confined inside the NW experiences a refractive index contrast at the end facets, thereby limiting the amount of reflection and feedback. As such, the threshold gain is a strong function of NW length and diameter.

It is known that the modal gain $g_{\mathrm{m}}$ is the product of the material gain $g_{0}$ and the optical confinement factor $\Gamma_{0}$ in conventional semiconductor lasers.

$$
g_{\mathrm{m}}=\Gamma_{0} g_{0} .
$$

Typically $\Gamma_{0}\left(0 \leq \Gamma_{0} \leq 1\right)$ describes the overlap between the gain region and the resonance mode, which is amplified within the cavity in a standard semiconductor laser with a weak waveguiding. This concept is no longer valid for NW lasers with high-index contrast or strong waveguiding. ${ }^{99-106}$ Mathematically, it has been proved that the confinement factor $\Gamma$ in a nanolaser, as displayed in Fig. 2(c), could be greater than $1,{ }^{106}$ hinting that the modal gain could be larger than the material gain. The physical meaning of this interesting phenomenon in nanolasers has also been clearly clarified in Ref. 106 where $\Gamma$ has been written as 


$$
\Gamma=\frac{v_{\mathrm{ph}}}{v_{\mathrm{E}}} \Gamma_{0},
$$

where $v_{\mathrm{ph}}$ is the phase velocity in the gain medium and $v_{\mathrm{E}}$ is the energy velocity of the waveguide. The slower energy velocity of the waveguide mode makes this prefactor more than 1 , yielding $\Gamma$ could be more than 1 . Most importantly, the larger modal gain makes it possible to have a laser with a lower threshold, which becomes very crucial in laser miniaturization.

Another major difference between NW and standard semiconductor lasers is their feedback or cavity mechanism. It is often assumed that a NW laser works as a FP cavity considering similar localized reflectors at both ends of the NW. But since the size of NW facets is comparable with or smaller than the wavelength involved, NW facets behave more like localized scattering centers rather than large planar reflectors considered in a standard FP cavity. Depending on the modes, wavelength, and diameter of the wires, the reflectivity in NWs can be significantly different from the results of the Fresnel formula for planar reflectors. ${ }^{107}$ Since the facet reflectivities determine the threshold of a laser, a good understanding and an accurate estimation of the facet reflectivities are very important and have been discussed in detail in recent publications. ${ }^{102,105,107}$

Modal characteristics of NWs can be analyzed by cylindrical waveguide theory. ${ }^{108}$ This is because the cylindrical NWs have transverse confinement structure similar to that of the optical fibers. Hence, the modal characteristics of NWs surrounded by air can be described by a full set of optical modes including transverse electric (TE), transverse magnetic (TM), and their hybrid modes (i.e., HE and EH modes) obtained from a cylindrical dielectric waveguide. Detailed analysis of modal characteristics in NW lasers can be found elsewhere. . $^{5,107,109,110}$

Like all nanoscale lasers, NW lasers exhibit more complicated far-field emission patterns than the conventional semiconductor lasers. As the size of lasers gets smaller, larger index contrast is required to obtain a better waveguiding. This leads to a divergent far-field profile as a result of fully vectorial (or nonparaxial) propagation. ${ }^{106}$ Besides, the scattered light output by the NW end facets further complicates the far field. Maslov and Ning ${ }^{107}$ theoretically analyzed the far-field emission patterns from individual NWs through a near-field scanning study. But the experiment was carried out on NWs horizontally lying on a substrate, which is different from the numerical simulation study. ${ }^{32}$ Further experiments on NWs, either vertically standing or suspended in air, are needed to verify the theoretical prediction. Moreover, a detailed theoretical understanding of the far-field behavior of a NW on a dielectric or a semiconductor substrate is required to compare with the experimental results. ${ }^{107}$

In most of the experimental realizations, III-nitride NW lasers are fabricated from homogeneous semiconductors, such as GaN, utilizing the simplest FP cavity. However, poor reflectivity with less than $25 \%$ at both top and bottom facets requires the NW to be as long as several tens of micrometers, in order to achieve lasing action. Due to the requirement of such unrealistically long NWs, achieving high-performance single NW lasers is fundamentally a technological challenge. Furthermore, on the way toward achieving wavelength tunable nanolasers, one could think of using InGaN embedded in NWs which could serve as a composition-variable gain medium. But the gain from the nanostructures with small volume is too low to overcome the cavity and high-mirror losses. Most importantly, significant scattering loss prevents smaller diameter NWs from lasing.

While recently reported dielectric optical microcavities, including microdisks, microposts, photonic crystals and metal claddings are approaching small-scale cavities, their physical sizes are larger than the emission wavelength for the effective confinement of photons. In contrast, plasmonics open up a new route for the strong confinement of light to a fraction of their freespace wavelength. Their unique dispersion relations enable propagation of light at short wavelengths along the metal-dielectric interfaces, making it possible to realize optical cavities with subwavelength mode volume and high-quality factor $(\mathrm{Q})$. This results in an enhanced lightmatter interaction and an increased spontaneous emission and strong coupling in quantum electrodynamics in such nanocavities. Furthermore, a giant modal gain can be achieved in such plasmonic nanocavity due to an even higher confinement factor defined in Eq. (5) for completely FP-based nanocavity. ${ }^{95}$ Importantly, the existence of this giant modal gain near the surface plasmon polariton (SPP) resonance will play an important role in achieving the smallest nanolasers; also due to the cost effectiveness and simplicity, the nanoplasmonic concept seems to 
be one of the most suitable approaches toward the realization of single NW lasers. For an optimized design of NW plasmonic laser, it is important to understand the basic operation of plasmonic FP nanolasers, which are presented elsewhere. ${ }^{111-113}$

\section{Growth of III-Nitride NWs}

Various methods have been developed for the growth and the synthesis of III-nitride NWs. The most commonly used methods for GaN NWs are chemical vapor deposition (CVD) and molecular beam epitaxy (MBE). NWs grown using these methods could have hexagonal, triangular, or rectangular cross-sectional areas, as displayed in Fig. 3. GaN NWs grown by metalorganic chemical vapor deposition (MOCVD) can exhibit either hexagonal or triangular cross-sections. ${ }^{14,115}$ High-quality InGaN NWs can also be grown by many methods including CVD ${ }^{81,116} \mathrm{MBE},{ }^{12,81,116-118}$ and hydride vapor phase epitaxy. ${ }^{119,120}$ Recently, significant efforts have already been devoted to the development of various InGaN NW heterostructures including core-shell, ${ }^{81,121}$ well/disk-in-a-wire, ${ }^{122,123}$ and dot-in-a-wire ${ }^{12,66}$ based nanostructures, wherein large bandgap $\mathrm{GaN}$ or $\mathrm{AlGaN}$ layers are often employed as the barrier or the shell structures to provide effective radial and/or axial carrier confinement. Currently, various InN nanostructures, such as nanocrystals, ${ }^{124}$ nanocolumns, ${ }^{125}$ nanorods, $,{ }^{126} \mathrm{NWs},{ }^{96}$ nanotubes, nanotips,${ }^{127}$ and nanobelts, ${ }^{36}$ have been extensively reported.

\section{Demonstration of III-Nitride Nanowire Lasers}

In the following sections, we will discuss the representative examples of experimentally demonstrated III-nitride NW lasers. The structural descriptions of each NW laser will also be briefly provided. These exemplary devices are classified based on their cavity configurations and feedback mechanisms employed.

\subsection{Fabry-Pérot Lasers}

In NW-based FP cavity, when the guided modes reach the NW end, part of them escape from the NW and the rest is reflected back to the cavity. The large refractive index difference between the semiconductor material and its surrounding dielectric environment enables photonic confinement in the NW cavities through the total internal reflection (TIR) mechanism, as shown in Fig. 4.

Significant efforts have already been undertaken by many research groups for using NW as a FP cavity for the realization of a laser. In 2002, two research groups from University of California, Berkeley, and Lawrence Berkeley National Laboratory, USA, achieved the first optically pumped room-temperature laser emission from a single GaN NW. ${ }^{32}$ The MOCVD-grown single GaN NW used in this study had lengths as long as $\sim 40 \mu \mathrm{m}$, in order to reduce the effective mirror loss.

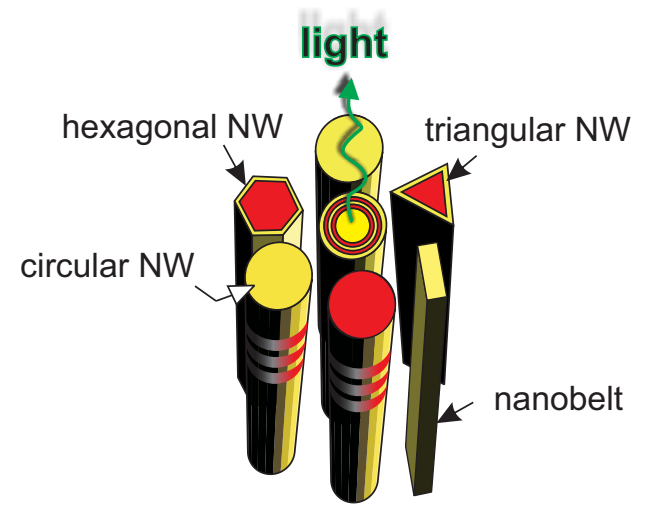

Fig. 3 Schematic structures of different types of NWs. 
(a)

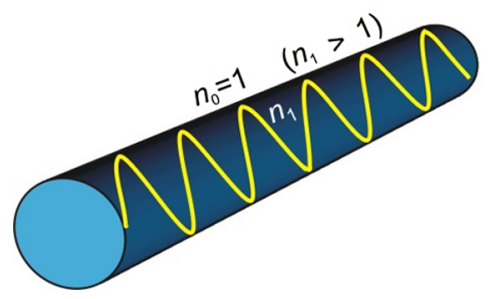

(b)

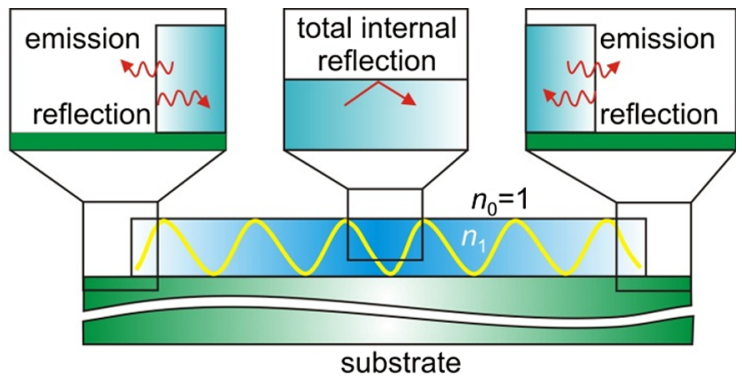

Fig. 4 (a) Schematic of a circular NW-based FP cavity. (b) Schematic of light propagation in a NW longitudinal cavity.

One year later, UV lasing from optically pumped $\mathrm{GaN} / \mathrm{Al}_{x} \mathrm{Ga}_{1-x} \mathrm{~N}$ core-sheath quantum wires was demonstrated by the same research group. ${ }^{23}$ Vapor-liquid-solid (VLS) epitaxial process was employed to grow cylindrical GaN cores with diameters as small as $5 \mathrm{~nm}$ cladded by a 50- to 100-nm layer of $\mathrm{Al}_{0.75} \mathrm{Ga}_{0.25} \mathrm{~N}$. Normally, GaN NWs with diameters less than $\sim 100 \mathrm{~nm}$ are too leaky to sustain laser cavity modes. Surrounding slender $\mathrm{GaN}$ wires with a material of larger bandgap and smaller refractive index creates a structure with simultaneous exciton and photon confinements. According to the authors, the core provides a gain medium, while the sheath acts as a FP cavity under optical pumping. Significantly, blueshifted PL, which is a signature of the quantum confinement effect, was observed from such unique nanostructures. But the lasing threshold was roughly 10 times higher than those of larger, unclad GaN NW lasers. ${ }^{32}$

In 2005, Gradečak et al. from Harvard University (USA) reported new optical studies of GaN NWs, which exhibited stimulated emission with lower lasing thresholds ${ }^{34}$ when compared with the results achieved in $2002 .{ }^{32}$ Such ultraviolet-blue emitting devices also operated at room temperature. The authors believed that NWs with improved structural characteristics led to excellent free-standing FP cavities, which, together with reduced deep-level defect-related emission by $n$-type doping, could further reduce the threshold pump power.

Again 1 year later, infrared lasing from single-crystalline InN NWs with belt geometry was demonstrated by research teams from Taiwan. ${ }^{36}$ Nanobelts employed into this study were grown by MOCVD and had well-faceted morphologies with atomically smooth surfaces. Such nanobelts had widths ranging from 40 to $250 \mathrm{~nm}$, thicknesses from 10 to $35 \mathrm{~nm}$, and length of $\sim 60 \mu \mathrm{m}$. Lasing action from such unique nanostructures was observed at $20 \mathrm{~K}$ under $\mathrm{CW}$ optical pumping.

A new type of tunable nanolaser was described by Qian et al. from Harvard University (USA) and Georgia Institute of Technology (USA) in $2008,{ }^{38}$ which involved the use of multiquantumwell (MQW) core/shell NW heterostructures. Lasing over a broad range of wavelengths at room temperature was reported here. In particular, the structure consists of a GaN NW core that acts as the optical cavity surrounded by InGaN/GaN MQW shells [see Fig. 5(a)] that serve as a composition-tunable gain medium. Such NW heterostructure contains 3 to 26 QWs. In this study, dimensions of NWs were 200 to $400 \mathrm{~nm}$ in diameter and 20 to $60 \mu \mathrm{m}$ in length. By varying the indium content, the emission wavelength can be tuned between 365 and $494 \mathrm{~nm}$, as shown in Fig. 5(b).

Recently, research groups from University of New Mexico, USA, and Sandia National Laboratory, USA, developed the first single-mode NW laser with a side-mode suppression ratio (SMSR) of more than $18 \mathrm{~dB}$ by using GaN NWs. ${ }^{46}$ Devices were optically pumped and lased at $370 \mathrm{~nm}$ with a threshold of $231 \mathrm{~kW} / \mathrm{cm}^{2}$. Then, the same research groups achieved a stable single-mode (SMSR $>15 \mathrm{~dB}$ ) operation in coupled GaN NW cavities utilizing Vernier effect as a mode-selecting mechanism. ${ }^{47}$ Such a coupled cavity was realized in this study by placing two NWs side-by-side in contact to form a NW pair, as displayed in Figs. 6(a) and 6(b). According to the authors, Vernier effect plays a major role in suppressing the multiple transverse-mode oscillation as well as multiple longitude-mode oscillation of the GaN NW, where its diameter is much larger than the wavelength. Devices were optically pumped and lased at $370 \mathrm{~nm}$ [Fig. 6(c)] with a threshold of $874 \mathrm{~kW} / \mathrm{cm}^{2}$.

Very recently, the same research groups together with researchers at the University of Essex, UK, have demonstrated a mechanism for lasing-mode selection by coupling a GaN NW laser to 

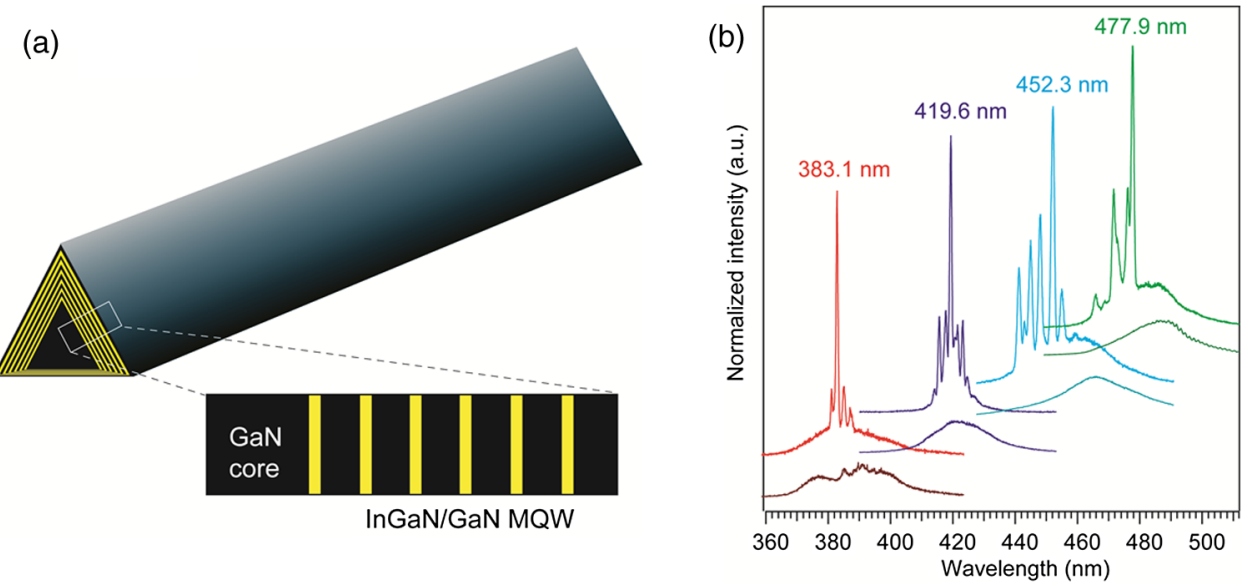

Fig. 5 (a) Schematic diagram of an InGaN/GaN MQW NW and magnified cross-sectional view of a NW facet highlighting the MQW structure. The InGaN layer is indicated in yellow color. (b) Normalized PL spectra collected from four representative $26 \mathrm{MQW}$ NW structures with increasing In composition pumped at 250 and $700 \mathrm{~kW} / \mathrm{cm}^{2}$, respectively. Spectra are offset for clarity. Reproduced with permission from Ref. 38.
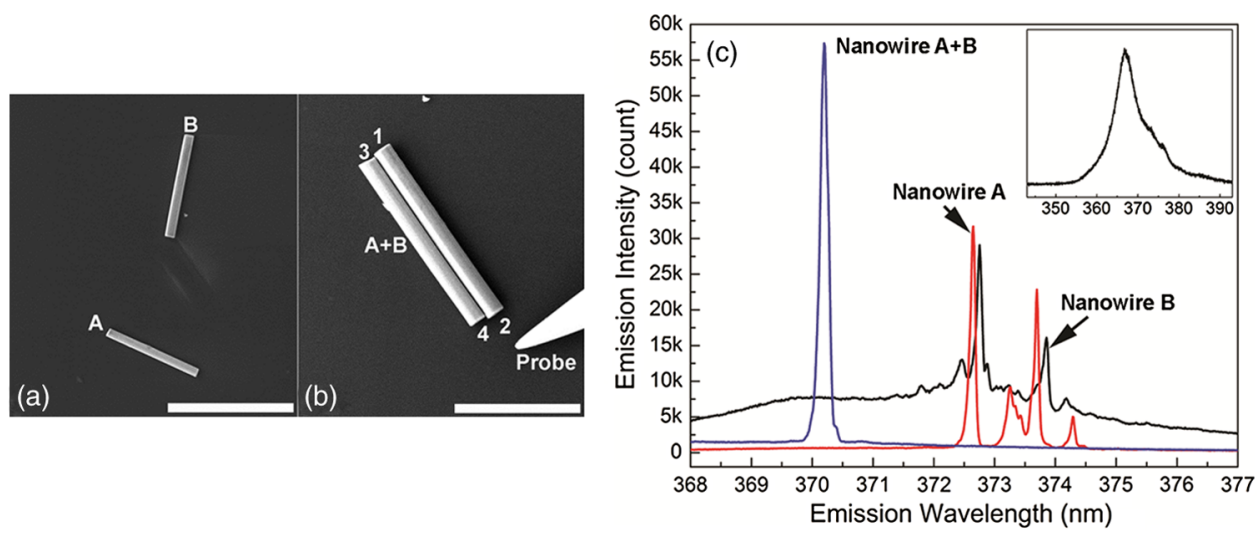

Fig. 6 (a) Individual GaN NWs. The scale bar represents $10 \mu \mathrm{m}$. (b) The GaN NWs were manipulated into a coupled NW cavity. The scale bar represents $5 \mu \mathrm{m}$. (c) The lasing emission spectra of the coupled NW pair and corresponding separated individual NWs. The three spectra were obtained with the same pump intensity of $1429 \mathrm{~kW} / \mathrm{cm}^{2}$. Inset: the PL spectrum of NW-A pumped below threshold. Reproduced with permission from Ref. 47.

an underlying gold substrate. ${ }^{48}$ A mode-dependent loss was induced by the gold substrate to suppress multiple transverse-mode operation, and thus, enable single-mode lasing behavior from NWs.

\subsection{Plasmonic Lasers}

One promising approach to realize a plasmonic nanolaser is the use of hybrid systems ${ }^{128}$ composed of noble metals and gain materials. Key to this design is the high-permittivity contrast at the low/high-dielectric interfaces, leading to a strong optical confinement in a thin (few nanometer) gap region, which greatly reduces plasmonic loss. ${ }^{128}$ The self-aligned single-crystalline NWs will be used as the gain medium in semiconductor nanolasers. Figure 7 displays the schematic of the plasmonic nanolaser consisting of a NW coupled to a crystalline thin metal film, e.g., $\mathrm{Ag}$, with a very thin insulating gap layer, e.g., $\mathrm{SiO}_{2}$. For the feedback mechanism, FP is introduced in the nanocavity where two end facets of the NW serve as mirrors. ${ }^{129}$

The first plasmonic III-nitride nanolaser based on such metal-oxide-semiconductor structure emitting in the green wavelength regime was realized by two research groups from National 


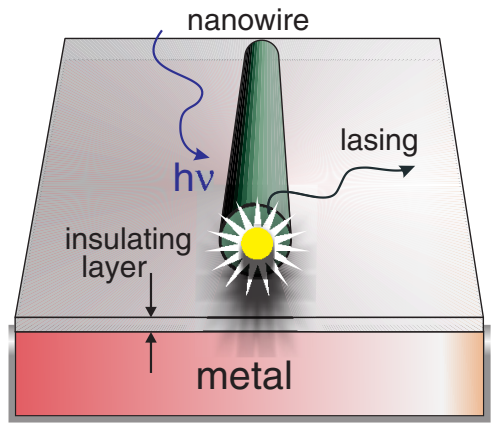

Fig. 7 Schematic of hybrid plasmonic waveguide-based laser.

Tsing-Hua University and National Chiao-Tung University, China. ${ }^{43}$ Optically pumped lasing has been demonstrated here with an operating temperature of $7 \mathrm{~K}$. Later, the same research groups along with some other collaborative groups from USA and China reported such hybrid plasmonic nanolasers with a better performance using the same concept but with some further refinement in the device structure. ${ }^{45}$ Devices with almost the same emission wavelength exhibited $\mathrm{CW}$ lasing operation up to $78 \mathrm{~K}$.

\subsubsection{Structural descriptions}

Wu et al. reported a nanoscale metal-oxide semiconductor (MOS) structure consisting of a greenemitting InGaN/GaN nanorod bundle coupled with a 50-nm thick colloidal gold triangular plate with a 5-nm thick spin-on-glass ( $\mathrm{SOG} ; \mathrm{SiO}_{2}$ ) gap layer. ${ }^{33}$ Later, $\mathrm{Lu}$ et al. refined such structures by replacing colloidal gold plate with epitaxially grown, atomically smooth $\mathrm{Ag}$ films as a scalable plasmonic platform. ${ }^{34}$ The NW plasmonic nanocavity was formed between epitaxial $\mathrm{Ag}$ film and a single nanorod consisting of a GaN shell and a green-emitting InGaN core as gain medium. On the top of the epitaxial $80-\mathrm{nm} \mathrm{Ag}$ thin film, the sample was capped with about $1.5 \mathrm{~nm}$ of amorphous germanium (Ge) grown by MBE. The nanocavity was defined by a 480-nm-long GaN nanorod partially filled with a 170 -nm-long $\mathrm{InGaN}$ gain medium and separated by a $5-\mathrm{nm} \mathrm{SiO}_{2}$ spacer from the epitaxial $\mathrm{Ag}$, as shown in Fig. 8(a). Note that in both studies, the InGaN/GaN core-shell nanorods were grown on $\mathrm{Si}(111)$ by plasmaassisted MBE with the nanorods growing along the wurtzite polar c-axis direction.

\subsubsection{Experimental results}

For the nanocavity with epitaxial Ag film, a light-light $(L-L)$ plot shows the output power of the lasing mode at $510 \mathrm{~nm}$ as a function of pump power for two different temperatures 8 and $78 \mathrm{~K}$ [Fig. 9(b)] under CW operation. The lasing signature can be seen from the concurrent onset of the linewidth narrowing plateau and the nonlinear kink of the "S"-shaped $L-L$ plot. The lasing thresholds are 2.1 and $3.7 \mathrm{~kW} / \mathrm{cm}^{2}$ at temperatures 8 and $78 \mathrm{~K}$, respectively. For a single

(a)

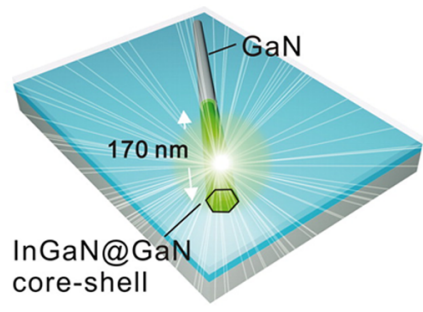

(b)

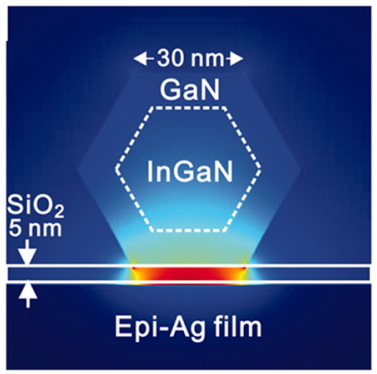

Fig. 8 (a) Schematic of a single InGaN/GaN core-shell nanorod on a $\mathrm{SiO}_{2}$-covered epitaxial $\mathrm{Ag}$ film. (b) The energy-density distribution is calculated by the eigenmode method. Reproduced with permission from Ref. 45. 

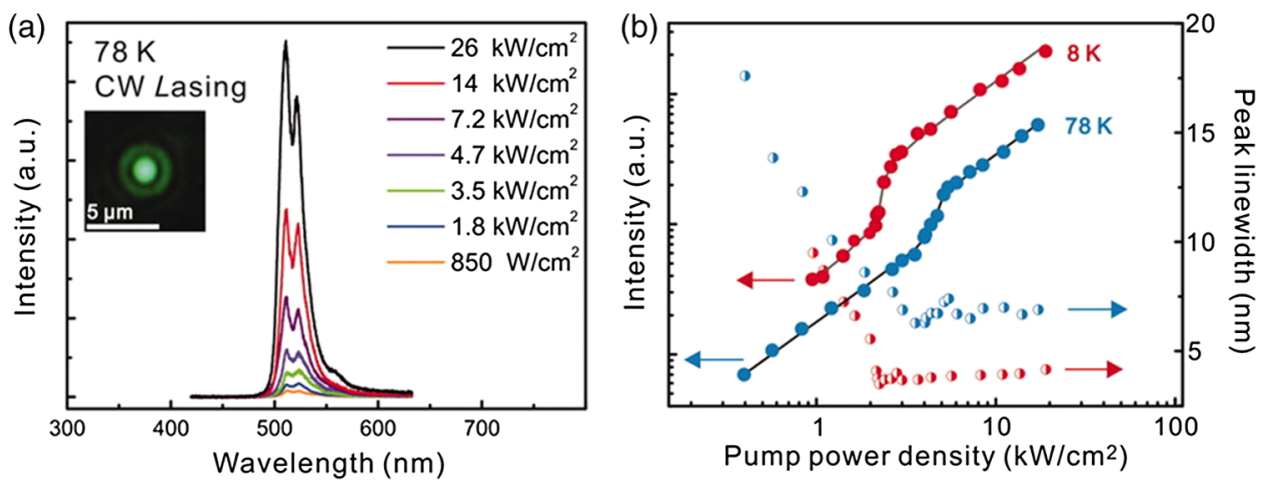

Fig. 9 (a) Lasing spectra for pumping by a CW 405-nm semiconductor diode laser. (Inset) The farfield laser spot with contrast fringes is a indicative of spatial coherence resulting from lasing. (b) Temperature-dependent lasing thresholds of the plasmonic cavity. The $L-L$ plots at the main lasing peak $(510 \mathrm{~nm})$ are shown with the corresponding linewidth-narrowing behavior measured at $8 \mathrm{~K}$ (red) and $78 \mathrm{~K}$ (blue) with lasing thresholds of 2.1 and $3.7 \mathrm{~kW} / \mathrm{cm}^{2}$, respectively. Reproduced with permission from Ref. 45.

nanorod, these power densities correspond to threshold powers of 56 and $100 \mathrm{nW}$ at the respective temperatures. Lu et al. claimed that the achieved CW-lasing thresholds for the Ag film-based plasmonic nanolasers are at least two orders of magnitude smaller when compared with their previously reported colloidal gold plate-based lasing structures with an emission wavelength of $533 \mathrm{~nm}$ under pulsed mode. ${ }^{33}$ This is mainly because of the use of atomically smooth epitaxial Ag film, which minimizes the plasmonic loss.

\subsection{Photonic Crystal Lasers}

While the modal gain of a NW FP cavity is strongly dependent on the NW length, ${ }^{130}$ an external high-Q photonic crystal microcavity providing a strong feedback regardless of the NW dimension offers the possibility of achieving nanoscale lasing. Taking this fact into account, an optically pumped monolithic single GaN NW laser on Si with a 2-D photonic crystal (PC) resonant cavity operating at room temperature has been recently reported by a group at the University of Michigan, Ann Arbor, Michigan. ${ }^{40}$

\subsubsection{Structural descriptions}

GaN NWs were grown on $\mathrm{Si}(111)$ substrates in the absence of a foreign metal catalyst in plasmaassisted MBE system. As schematically shown in Fig. 10(a), the device heterostructure consists of a single GaN NW at the center of a H2 defect and surrounded by a 2-D PC microcavity fabricated using $\mathrm{TiO}_{2}$ layer and spin-on-glass (SOG). Here, the SOG layer, a low refractive index material, was chosen to reduce the optical loss from the $\mathrm{TiO}_{2}$ layer to the $\mathrm{Si}$ substrate. The PC was arranged in a triangular lattice with air holes of radius $40 \mathrm{~nm}$ and period $120 \mathrm{~nm}$. During device fabrication, a small hollow was formed around the NW in the center of the $\mathrm{H} 2$

(a)

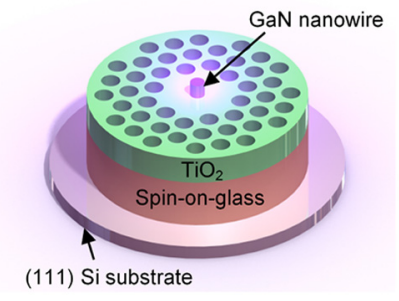

(b)

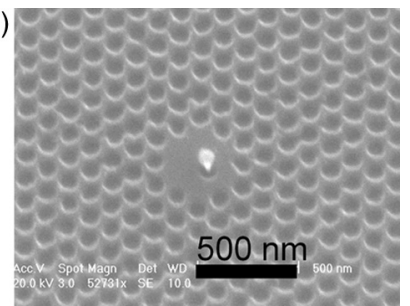

Fig. 10 (a) Schematic representation of NW laser consisting of a single GaN NW and a 2-D photonic crystal microcavity. (b) An oblique view scanning electron microscopy (SEM) image of the fabricated device. Reproduced with permission from Ref. 40. 

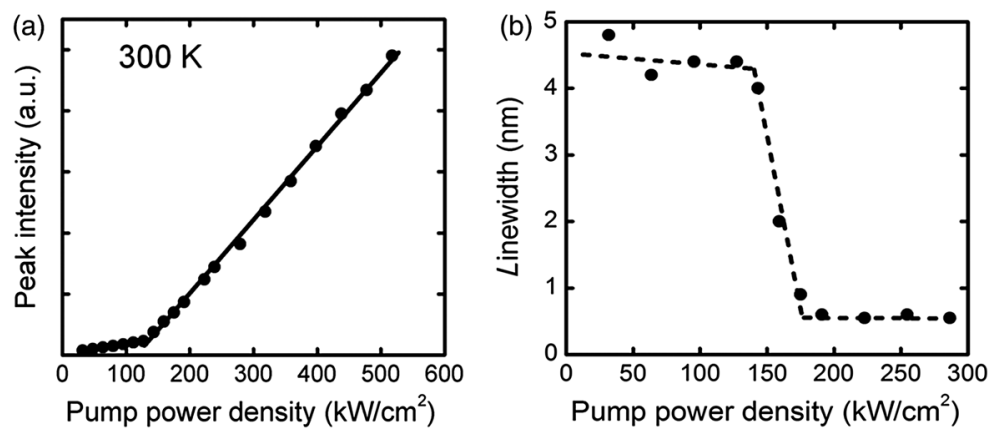

Fig. 11 (a) Variation of peak output intensity with pump power (the $L-L$ curve). The change in slope near threshold $\left(\sim 120 \mathrm{~kW} / \mathrm{cm}^{2}\right)$ is clearly observed. (b) Measured linewidth of the dominant peak as a function of the pump power density. Reproduced with permission from Ref. 40.

defect. Based on the calculation, such hollow was assumed to be a partially filled hole with a diameter of $80 \mathrm{~nm}$, whereas its depth was roughly estimated to be $\sim 30 \mathrm{~nm}$ based on the amount of $\mathrm{TiO}_{2}$ deposited on the top of the NW.

\subsubsection{Experimental results}

The authors reported the signature of laser action in such PC structure under optical excitation with a threshold pump power densities of $\sim 120 \mathrm{~kW} / \mathrm{cm}^{2}$, as illustrated in Fig. 11(a). The device was operated at room temperature under quasi-CW mode. Moreover, the output emission exhibited a fairly narrow peak with a linewidth of $4.5 \mathrm{~nm}$ at $370.4 \mathrm{~nm}$. According to the authors, this could be due to the Purcell effect, which leads to an enhanced spontaneous emission. Such enhanced emission becomes even stronger near threshold and turns into a coherent laser-like emission above threshold. The variation of the measured emission linewidth with pump power is shown in Fig. 11(b). The linewidth falls off very rapidly after the device reaches its threshold indicating the onset of lasing.

\subsection{Ring Resonator Lasers}

Recently, ring resonators are of significant research interest as building blocks for various photonic applications including wavelength division multiplexers, add-drop filters, and lasers. Compared with other kinds of cavities, ring resonators offer scalability in size and flexibility in mode design. Moreover, a ring cavity offers a better resonance than a linear FP cavity because of the efficient recirculation of light. ${ }^{131,132}$

\subsubsection{Structural descriptions}

Nitride ring resonator nanolasers were first fabricated and analyzed by a Berkeley group led by Yang. ${ }^{35}$ Nanoring resonator is formed by converting a NW into a ring. The ring structure fabricated from a linear GaN NW was synthesized via a chemical vapor transport process in a hot-wall furnace with metallic gallium and ammonia precursors. Single [11̄0] growth-direction wires with triangular cross-sections were modified with the micromanipulator by pushing the two ends together in a side-by-side geometry to ensure optical coupling, as shown in Fig. 12.

\subsubsection{Experimental results}

Wire and ring structures, as displayed in Figs. 13(a) and 13(b), respectively, were optically pumped by varying the excitation power under pulsed operation. Both structures exhibited lasing action at room temperature. There is a clear change in the lasing behavior of the ring structure as compared with the linear NWs. A significant redshift relative to the linear wire is observed in such ring structures. For example, under similar power fluence $\left(1050 \mu \mathrm{J} / \mathrm{cm}^{2}\right)$, the ring nanolaser in Fig. 13(c) has its maximum emission intensity which is 9-nm redshifted from 


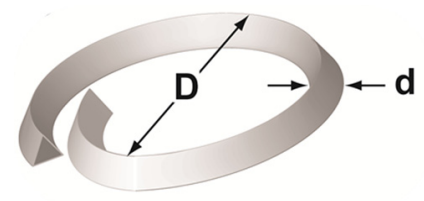

Fig. 12 Schematic of ring structures showing the triangular cross-section of the NWs and the sideby-side overlap that enables evanescent coupling between cavity arms. $D$ is the ring diameter and $d$ is the NW diameter. Reproduced with permission from Ref. 35.

the equivalent linear structure. Please note that the measured threshold power density of such ring structure is $112.5 \mu \mathrm{J} / \mathrm{cm}^{2}$, being $50 \%$ higher than its linear counterpart. According to the authors, changes to the optical boundary conditions of the lasing cavity affect the structure's PL, photon confinement, and lasing as a function of the ring diameter. Unlike linear FP cavity, the ring resonator nanolaser exhibits a significant redshift with the change of the ring diameter due to conformational changes of the cavity. These results clearly demonstrated the versatility of NW laser cavities for using as tunable laser systems in future integrated-photonic platforms.

\subsection{Microstadium Lasers}

Recently, there has been much interest generated in a stadium-shaped microcavity which is rigorously known to be fully chaotic. Such stadium-shaped laser diodes were pioneered by Siegman's group, and the oscillation behaviors have been studied for this type of resonator. ${ }^{133}$ A stadium microresonator is particularly interesting for the following reasons:

- One problem for semiconductor microcavity lasers is nonradiative recombination of injected carriers via surface electronic states. In order to reduce this detrimental effect, a stadium-shaped chaotic microcavity is generally utilized which pulls the resonance modes away from the boundary. Here, the cavity shape is deformed from circles, so that the lasing modes are no longer whispering-gallery (WG) modes.

- Unlike a circular microcavity, in a microstadium cavity even the carriers near the cavity center can contribute to lasing of the WG modes. This is due to the fact that a microstadium cavity pushes the resonating modes to the interior of the cavity. As a result, carriers at the cavity center can also be utilized and contribute to lasing gain. Such an effective carrier utilization process is more important for electrically-pumped devices, wherein carriers are usually injected to the central region of a microcavity.

- Chaotic microstadium cavity could produce directional output beams.

- Output power from such resonators is also high, due to the reduced evanescent leakage of laser light.
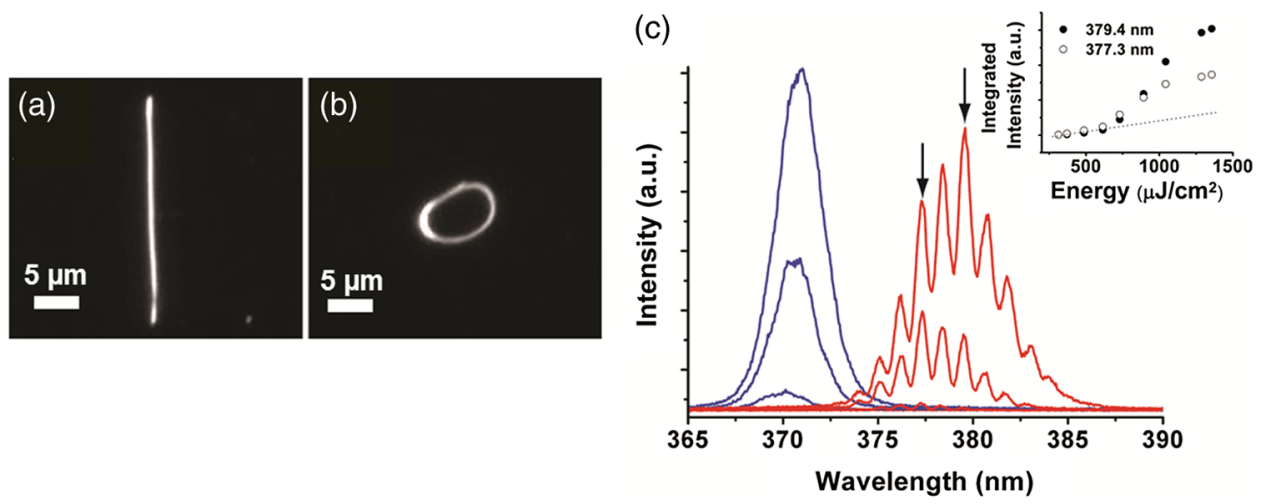

Fig. 13 (a) A linear GaN NW before physical manipulation of it into a ring configuration. (b) Corresponding ring geometry made from the wire in (a); scale bar $=5 \mu \mathrm{m}$ in both cases. (c) Lasing spectra for the wire (dark blue) and ring (light red) structures. Arrows correspond to integrated modes within the inset. Inset: modal gain analysis for two separate modes from the ring resonator cavity. Reproduced with permission from Ref. 35. 
In spite of all the above-mentioned advantages, the chaotic microcavity lasers usually exhibit a low-Q which leads to a high lasing threshold. The first microstadium III-nitride NW laser reported by Park et al. was a hybrid approach that combined an active semiconductor NW with a stadium microresonator for low-threshold stimulated emission. ${ }^{37}$ Here room-temperature lasing in an optically pumped single NW stadium microresonator emitting at $372 \mathrm{~nm}$ was reported.

\subsubsection{Structural descriptions}

For the realization of microstadium laser, the hybrid structure developed by the collaborators from Korea University and Harvard University consists of a single-crystal GaN NW and a silicon nitride $\left(\mathrm{Si}_{3} \mathrm{~N}_{4}\right)$ stadium microresonator. ${ }^{37}$ The $\mathrm{Si}_{3} \mathrm{~N}_{4}$ slab structure was fabricated on a thick $\mathrm{SiO}_{2}$ post with a low refractive index of $1.5 . \mathrm{Si}_{3} \mathrm{~N}_{4}$ is essentially transparent and exhibits a high refractive index of 2.1 at the emission wavelength of $365 \mathrm{~nm}$, corresponding to the band gap energy of $\mathrm{GaN}(\sim 3.4 \mathrm{eV})$. Hence, $\mathrm{Si}_{3} \mathrm{~N}_{4}$ structure establishes a microstadium optical cavity surrounding the NW, as shown in Fig. 14(a).

Figure 14(b) shows the SEM image of a fabricated stadium microresonator using a NW. The $\mathrm{NW}$ on the top of the stadium was $\sim 7.5 \mu \mathrm{m}$ long. The length of one side of such NWs with triangular cross-section was $\sim 170 \mathrm{~nm}$. Relatively thin and short NWs were used in this study to minimize the cavity effect of NW itself. The magnified SEM image of a NW end is shown in the inset of Fig. 14(b). NWs are placed on the $\mathrm{Si}_{3} \mathrm{~N}_{4}$ slab in a way that their two ends are aligned and located at the two foci of such microstadium.

\subsubsection{Experimental results}

Figure 15(a) displays the PL peak intensities of the microstadium structure as a function of pump power density. It is shown that above a threshold power density of $1536 \mathrm{~kW} / \mathrm{cm}^{2}$, the PL intensities clearly show a sharp transition from spontaneous to stimulated emission. Figure 15(b) shows the lasing-mode image measured in the structure. Since the refractive indices of GaN and $\mathrm{Si}_{3} \mathrm{~N}_{4}$ are very close to each other at the wavelength of $365 \mathrm{~nm}$, light is coupled from the NW with the $\mathrm{Si}_{3} \mathrm{~N}_{4}$ slab. Moreover, light emission from the NW can be strongly confined in the entire $\mathrm{Si}_{3} \mathrm{~N}_{4}$ stadium, as shown in Fig. 15(b). It should be noted that the measured threshold of this microstadium laser is larger than the threshold of a typical single NW laser on glass which is on the order of 100 to $1000 \mathrm{~kW} / \mathrm{cm}^{2}$. But these research groups believe that even higher confinement factor without sacrificing the high-Q can be achieved in the hybrid single NW structure by using higher refractive index microstadium and/or lower refractive index substrate. Thus, the laser threshold in the device could be further reduced.

\subsection{Random Lasers}

Optical scattering was often considered to be detrimental to laser performance, because such scattering removes photons from the lasing modes of a conventional laser cavity. However, it is found that light scattering plays a positive role in a disordered active medium and can provide an effective means of achieving gain for laser oscillation. Multiple scattering increases the
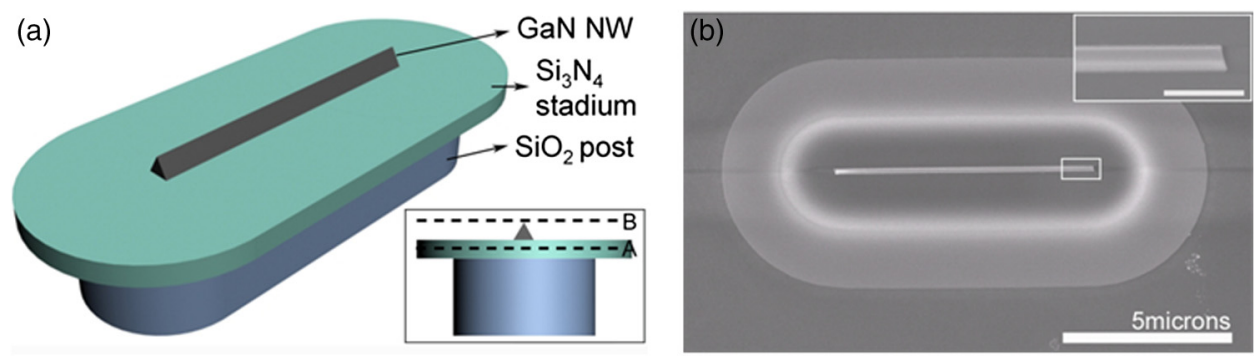

Fig. 14 (a) Schematic illustration of NW stadium microresonator. Inset: sideview of the structure. (b) SEM image of a fabricated NW stadium microresonator. Inset: magnified SEM image of a NW end (scale bar is $500 \mathrm{~nm}$ ). Reproduced with permission from Ref. 37. 

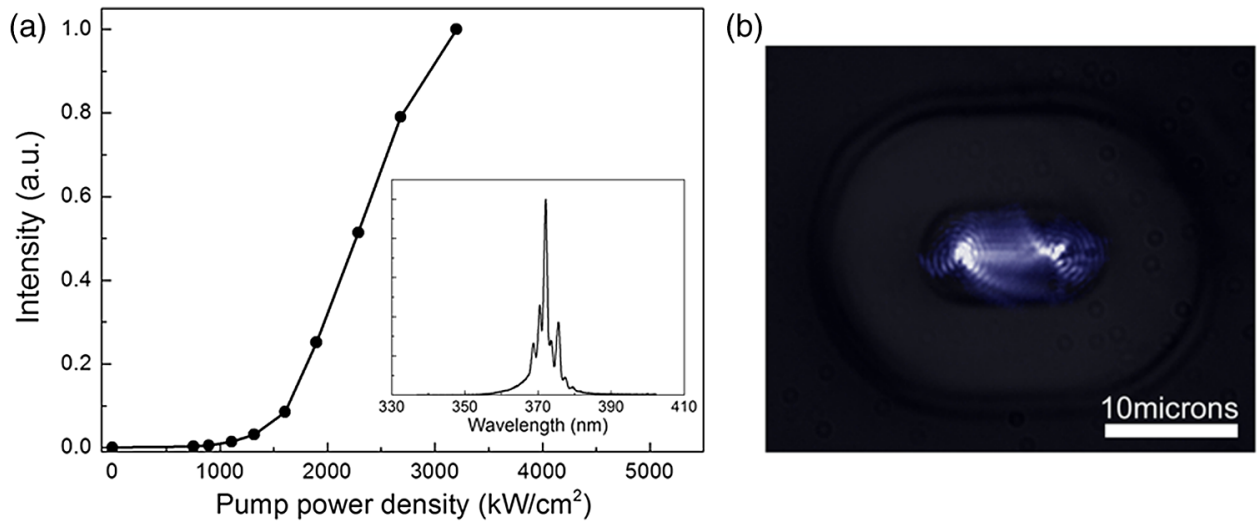

Fig. 15 (a) Lasing peak intensity versus pump power density. Inset: PL spectrum at $2287 \mathrm{~kW} / \mathrm{cm}^{2}$. (b) PL image (false-color) superimposed on white-light image (grayscale). The pump power density is $2680 \mathrm{~kW} / \mathrm{cm}^{2}$. Reproduced with permission from Ref. 37.

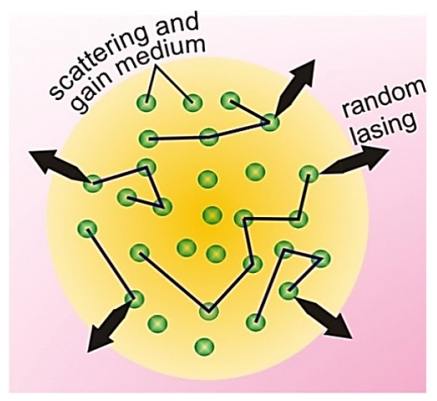

Fig. 16 Schematic of a random laser, where the cavity is absent but multiple scattering between particles in the disordered material keeps the light trapped long enough for the amplification to become efficient and for laser light to emerge in random directions.

optical path length in a gain medium which results in enhanced laser amplification as illustrated in Fig. 16. In addition, recurrent light scattering could provide coherent feedback for laser oscillation. ${ }^{134}$ Recently, there are great interests in studying photonic quasicrystals, which may lie somewhere between periodic and random structures. Utilizing such quasi-periodic structures, one could think of designing a pseudo-random laser with deterministic properties, which may play a significant role for practical applications. In a related approach, Chang et al. recently reported the observation of stimulated emission resembling random lasing from optically pumped GaN quasicrystal nanorod arrays. ${ }^{44}$

\subsubsection{Structural descriptions}

The quasicrystal nanorod array was formed on MOCVD-grown 3- $\mu$ m-thick GaN epitaxial layer. Using nanoimprint lithography, top-down patterned etching was done on this layer to prepare a 12-fold symmetric quasicrystal pattern. Crystalline facets were formed on the etched GaN nanorod surfaces. MOCVD regrowth was done to grow multiple InGaN/GaN QWs on the crystalline facets, which led to a core-shell nanorod structure. The plane-view SEM image of the fabricated nanorods is shown in Fig. 17(b), which shows the 12-fold symmetric quasicrystal pattern of nanorod arrays. The sidewall length of hexagonal nanorod is $\sim 360 \mathrm{~nm}$. The quasicrystal pattern shown in Fig. 17(a) is overlaid on the SEM image to show its quasicrystal pattern.

\subsubsection{Experimental results}

The PL spectra of nanopillar sample at various pump intensities are shown in Fig. 18(a), where the legends are pump intensities indicated in Fig. 18(b). With increasing pumping intensity, multiple emission peaks with narrow linewidths in the range of 0.2 to $0.3 \mathrm{~nm}$ appeared 

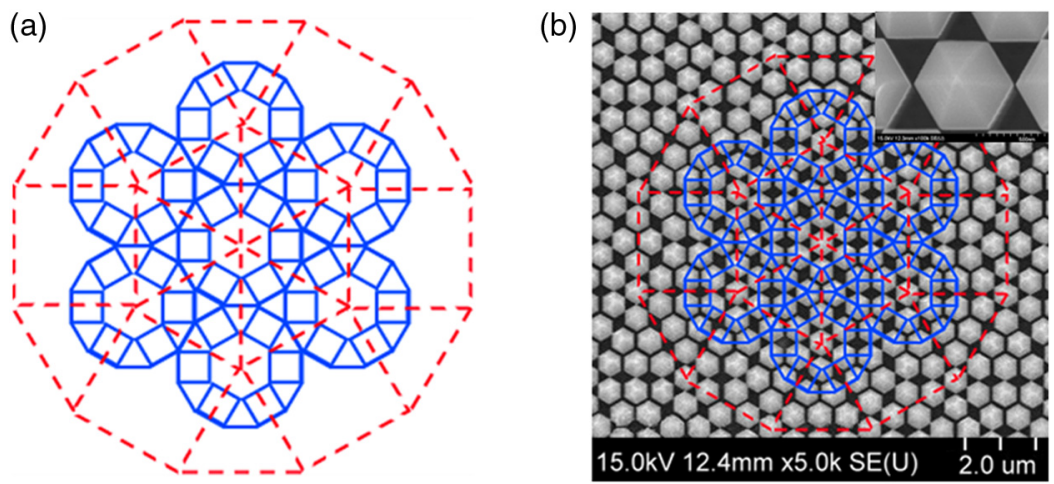

Fig. 17 (a) 12-fold symmetric quasicrystal pattern. (b) SEM plane-view image of the fabricated GaN quasicrystal nanorod arrays. The upper right inset image shows the crystalline facets. Reproduced with permission from Ref. 44.
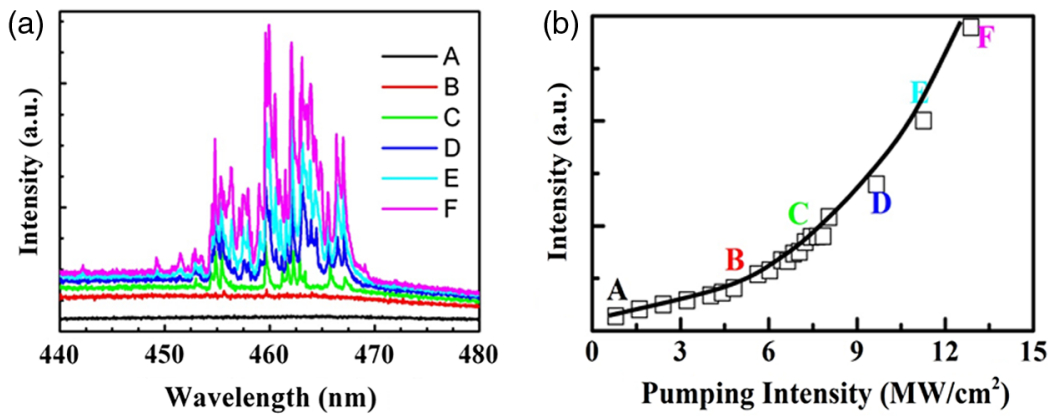

Fig. 18 (a) PL spectra at various pump power densities labeled in graph (b). (b) The integrated intensity versus pump intensity. Reproduced with permission from Ref. 44.

from the broad emission background. The integrated intensity within such broad emission background ranging from 455 to $470 \mathrm{~nm}$ as a function of pump intensity is shown in Fig. 18(b), where an onset of lasing action at threshold pump intensity of $\sim 5 \mathrm{MW} / \mathrm{cm}^{2}$ is clearly observed. Please note that this broad emission wavelength range corresponds to the high gain regime of the MQWs embedded in nanorods. Unlike conventional lasers, there is an obvious irregularity for the emission wavelengths (or the spacing among them) of these multiple lasing peaks. It is unlikely that these irregular lasing modes are due to WG modes of individual nanorods or any regular optical feedback paths. Another important point worth noting is that the intensities of these lasing peaks do not necessarily show the increasing trend with pump power. The intensities for some of these peaks may even decrease at some point with increasing power, when compared with other emerging peaks, hinting that there are mode competitions among lasing peaks.

\subsection{Polariton Lasers}

Polaritons are light-matter hybrids which form through interactions between highly confined light and an excited electron-hole pair (exciton) within a cavity. Unlike conventional photon lasers, polariton lasers are not constrained by population inversion and stimulated emission, i.e., very little energy is required to operate a polariton laser. Therefore, it is strongly believed that polaritons offer a way to create lasers with very low power requirements. Furthermore, polariton lasers will bring fundamental benefits of many-body quantum physics in our reallife applications. Their unique physical properties could be utilized for realizing novel types of application-suited spin switches and terahertz lasers. ${ }^{135}$ One of the most promising materials for polariton lasing is $\mathrm{GaN}$, which allows for room-temperature action due to a large exciton binding energy. Recently, a new structure containing GaN NWs as the active media was 
developed by a group at the University of Michigan in Ann Arbor, Michigan, to fabricate polariton lasers. ${ }^{42}$ Such lasers with an emission wavelength of $\sim 370 \mathrm{~nm}$ operate at room temperature with an ultralow threshold.

\subsubsection{Structural descriptions}

For the purpose of demonstrating a polariton laser, the authors used a single GaN NW in a dielectric microcavity. As shown schematically in Fig. 19, the NW was buried in a $\lambda$-thick inner cavity made of $\mathrm{SiO}_{2}$. This inner cavity was sandwiched between a top and a bottom dielectric Bragg mirror made of seven pairs of $\mathrm{SiO}_{2} / \mathrm{TiO}_{2}$. A single NW was placed at the central antinode of the cavity field so that the interaction between the GaN NW excitons and the cavity mode became maximized. The inset of Fig. 19 shows the SEM image of a horizontally lying NW on the top of the half cavity, which is without the top cavity. Such buried cylindrical NW with a diameter of $\sim 60 \mathrm{~nm}$ was $\sim 750 \mathrm{~nm}$ long.

\subsubsection{Experimental results}

The authors recorded a sharp increase of the PL intensity from lower polaritons (LPs) at in-plane wave number $k_{\|} \sim 0$ with increasing pumping power, as shown in Fig. 20(a). The incident excitation energy at threshold was $E_{\mathrm{th}}=92.5 \mathrm{~nJ} / \mathrm{cm}^{2}$ at $300 \mathrm{~K}$. Such behavior was confirmed by a sharp decrease in the emission linewidth and a small blueshift of the LP energy [Fig. 20(b)]. These two features are clear indications of stimulated scattering into the LP states near $k_{\|}=0$. A second distinct threshold relating to a conventional photon laser was found at $E_{\mathrm{th} 2}=2700$ and $E_{\text {th } 1}=250 \mu \mathrm{J} / \mathrm{cm}^{2}$. In other words, the second threshold corresponding to photon lasing was observed at a density of 2700 times the polariton lasing threshold density. According to the authors, the threshold density in such a device was 2 orders of magnitude lower than any previously reported GaN-based polariton lasers.

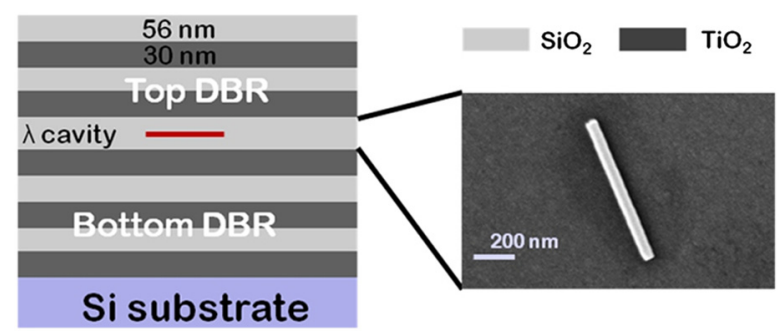

Fig. 19 Schematic representation of the dielectric microcavity with a single GaN NW of a diameter $60 \mathrm{~nm}$ (inset) buried in the center of a $\lambda$-thick cavity. Reproduced with permission from Ref. 42.

(a)

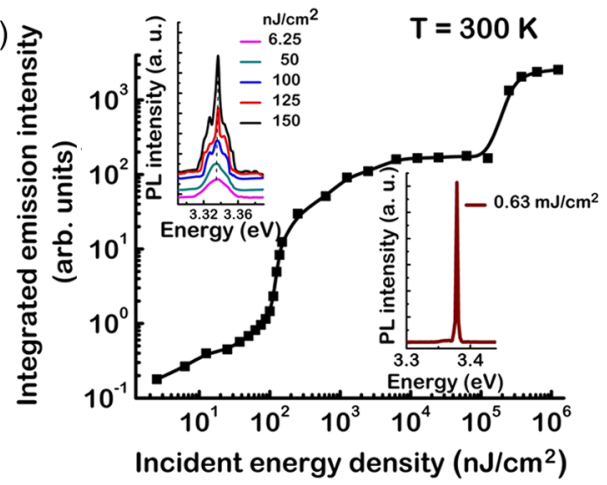

(b)

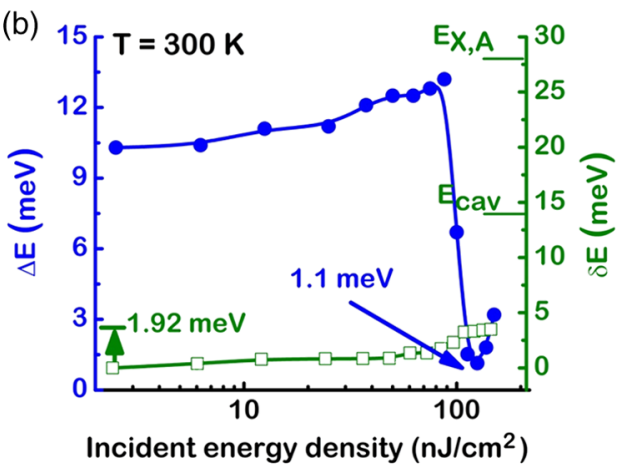

Fig. 20 (a) Variation of the emission intensity with excitation energy at $300 \mathrm{~K}$ clearly showing the two thresholds for polariton and photon lasing. The insets show the corresponding emission spectra. (b) Variations of linewidth and emission energy at $300 \mathrm{~K}$ for polariton lasing. Reproduced with permission from Ref. 42. 


\subsection{Two-Dimensional DFB Lasers}

Since the first introduction of distributed-feedback (DFB)-based laser by Kogelnik and Shank, ${ }^{136}$ this concept has undergone rapid developments, in order to obtain lasing action. The fabrication of 2-D-DFB GaInAsP/InP laser diodes was one of the greatest achievements, in which the laser beam was radiated perpendicularly to the surface. ${ }^{137}$ The same concept has been applied to the study of blue-violet GaN 2-D-DFB laser action, where the periodic air-hole structures inducing the 2-D light confinement was used. ${ }^{138}$ Recently, Kouno et al. from Sophia University, Japan, first experimentally demonstrated high-performance 2-D-DFB laser action in the blue wavelength regime using high-density InGaN/GaN nanocolumn array having a dielectric cylinder. ${ }^{39}$ On the way toward the realization of lasers with green emission wavelength, later Ishizawa et al. reported stimulated emission as 2-D-DFB lasers by employing InGaN-QW nanocolumn arrays. $^{41}$

\subsubsection{Structural descriptions}

Kouno et al. used a MOCVD-grown (0001) 3.5- $\mu \mathrm{m}$ thick GaN template, whereas GaN rectangular-lattice nanocolumn arrays with 8-period InGaN/GaN MQWs at the top of the columns were grown by RF-MBE. ${ }^{39}$ Figure 21 displays SEM images of a vertically aligned nanocolumn array sample fabricated in this study. Each GaN nanocolumns with a hexagonal cross-section had the side length of $92 \mathrm{~nm}$ and the height along the c-axis of approximately $850 \mathrm{~nm}$. The GaN nanocolumns were arranged in a rectangular lattice by adjusting a particular horizontal and vertical lattice constant [see Fig. 21(b)]. Most importantly, the lasing wavelength of a nanocolumn array is controlled by two parameters, i.e., lattice constant and hexagonal side length.

\subsubsection{Experimental results}

Figure 22(a) displays the RT emission spectra measured by changing the excitation power intensity, where a sharp and a strong emission peak was observed at $471 \mathrm{~nm}$ at a power density of approximately $320 \mathrm{~kW} / \mathrm{cm}^{2}$. The intensity increased nonlinearly as a function of excitation power density, as shown in Fig. 22(b). This was the clear signature of the stimulated emission. The 2-D light confinement enhanced the optical gain at this wavelength, ${ }^{139}$ yielding the stimulated emission. Using the similar concept, Ishizawa et al. demonstrated blue to green stimulated emission from InGaN-QW nanocolumn arrays, in which the emission wavelength was controlled through the structural parameters of the lattice constant and the nanocolumn diameter in the wavelength ranges of 440 to 510 and 530 to $560 \mathrm{~nm}$, respectively. ${ }^{41}$ Note that the lasing threshold for the nanocolumn arrays with a peak emission wavelength of $552 \mathrm{~nm}$ appeared at excitation power densities greater than $0.42 \mathrm{MW} / \mathrm{cm}^{2}$. The authors claimed that $560 \mathrm{~nm}$ was the longest wavelength ever reported for stimulated emission among InGaN-based QWs.
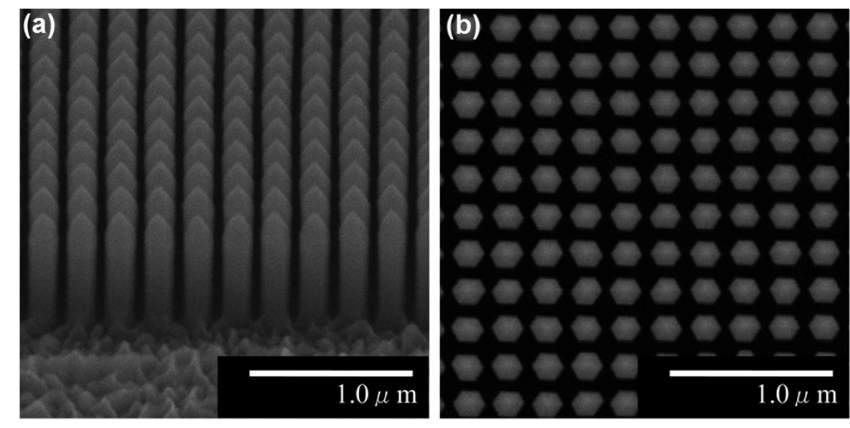

Fig. 21 SEM images: (a) bird's-eye and (b) top views of typical regularly arranged GaN nanocolumns with 8-period InGaN/GaN MQWs. Reproduced with permission from Ref. 39. 

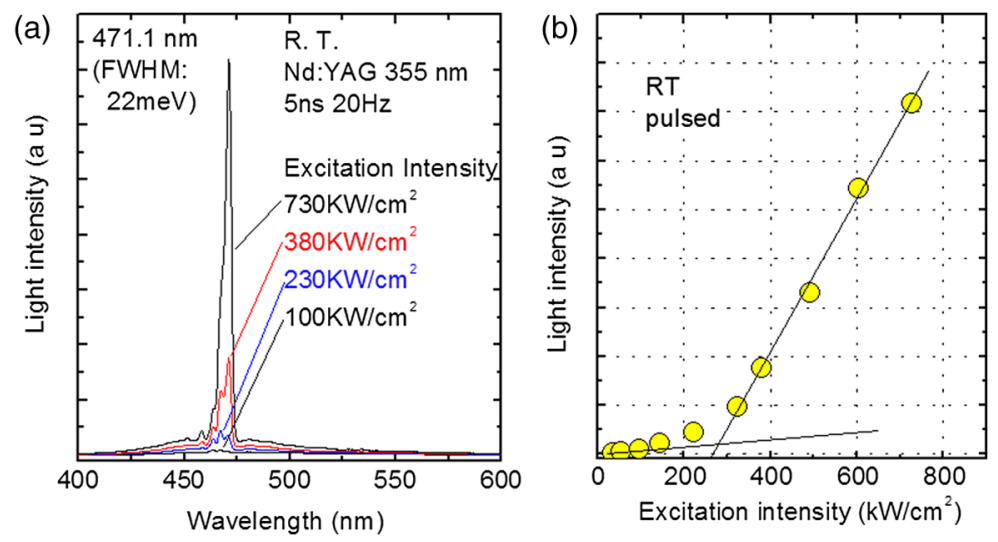

Fig. 22 (a) High-excitation RT-PL spectra and (b) dependence of RT-PL emission peak intensity of an InGaN-based nanocolumn array. The emission light was polarized in TE mode. Reproduced with permission from Ref. 39.

\section{Future Directions and Conclusion}

In summary, we have provided a detailed overview of the current research status of III-nitride NW lasers. In spite of the achievements of optically pumped nanolasers worldwide, there have been no results on electrically pumped III-nitride NW lasers reported so far. Such electrically driven semiconductor nanolasers are required for practical applications. There are several issues that need to be addressed, in order to achieve electrical injection in nanolasers. From the growth point of view, nearly defect-free NWs with well-controlled doping levels as well as active regions with sufficiently large gain will be needed. From the viewpoint of fabrication, it is necessary but challenging to obtain a low-resistive metal-semiconductor Ohmic contact at the nanoscale level without degrading the optical confinement. Many device physics issues such as electrical and thermal transport properties, carrier injection efficiency, and lateral band bending due to surface defects or morphology in such nano- or sub-micron scale structures need to be understood through detailed experimental and theoretical studies. Meanwhile, various $p-i-n$ heterostructures in axial or radial direction or in cross-bar configuration ${ }^{23,28,38}$ have been fabricated, but it is still not clear which approach provides better electrical injection and what the optimized structures are for efficient carrier injection.

Nanolasers with reduced threshold and large Purcell factors open a fundamentally new pathway for improving laser modulation bandwidth. ${ }^{140}$ In spite of the recent demonstration of optically pumped photonic crystal nanocavity lasers with thresholds of only a few nanowatt ${ }^{141}$ and modulation rates exceeding $100 \mathrm{GHz},{ }^{142}$ to our best knowledge, there have been no reports on the dynamic characteristics of NW-based lasers. It is, therefore, also of interest to explore III-nitride NW lasers for high-speed modulation that is relevant for communication applications.

For most real-world applications, the output power needs to be reasonably high, which, to date, has not been obtained from NW lasers. It is believed that by addressing several issues such as improving the carrier injection efficiency, optimized heterostructures, cavity loss minimization, and effective thermal management, the output power of NW lasers can be drastically increased. In addition, mode control of nanolasers including single-mode operation in such NW lasers needs to be examined. The development of such high-performance III-nitride NW lasers that can operate in the near-infrared, visible, and deep UV spectral ranges is of immense interest, especially for optical communications, quantum computing, display, lighting, biosensing, medical, and data storage applications.

\section{References}

1. R. S. Wagner and W. C. Ellis, "Vapor-liquid-solid mechanism of single crystal growth," Appl. Phys. Lett. 4(5), 89-90 (1964), http://dx.doi.org/10.1063/1.1753975. 
2. R. S. Wagner, Whisker Technology, Wiley-Interscience, New York (1970).

3. X. Y. Bao et al., "Heteroepitaxial growth of vertical GaAs nanowires on $\mathrm{Si}(111)$ substrates by metal-organic chemical vapor deposition," Nano Lett. 8(11), 3755-3760 (2008), http:// dx.doi.org/10.1021/n1802062y.

4. S. Li and A. Waag, "GaN based nanorods for solid state lighting," J. Appl. Phys. 111(7), 071101 (2012), http://dx.doi.org/10.1063/1.3694674.

5. S. Zhao et al., "Growth of large-scale vertically aligned GaN nanowires and their heterostructures with high uniformity on $\mathrm{SiO}(\mathrm{x})$ by catalyst-free molecular beam epitaxy," Nanoscale 5(12), 5283-5287 (2013), http://dx.doi.org/10.1039/c3nr00387f.

6. X. Duan et al., "Indium phosphide nanowires as building blocks for nanoscale electronic and optoelectronic devices," Nature 409(6816), 66-69 (2001), http://dx.doi.org/10.1038/ 35051047.

7. Y. Cui and C. M. Lieber, "Functional nanoscale electronic devices assembled using silicon nanowire building blocks," Science 291(5505), 851-853 (2001), http://dx.doi.org/10 .1126/science.291.5505.851.

8. Y. Dong et al., "Coaxial group III-nitride nanowire photovoltaics," Nano Lett. 9(5), 21832187 (2009), http://dx.doi.org/10.1021/n1900858v.

9. Y. Huang, X. Duan, and C. M. Lieber, "Nanowires for integrated multicolor nanophotonics," Small 1(1), 142-147 (2005), http://dx.doi.org/10.1002/smll.200400030.

10. A. K. Wanekaya et al., "Nanowire-based electrochemical biosensors," Electroanalysis 18(6), 533-550 (2006), http://dx.doi.org/10.1002/(ISSN)1521-4109.

11. E. Wong et al., "Energy-efficiency of optical network units with vertical-cavity surfaceemitting lasers," Opt. Express 20(14), 14960-14970 (2012), http://dx.doi.org/10.1364/OE .20 .014960 .

12. Y. L. Chang et al., "High efficiency green, yellow, and amber emission from InGaN/GaN dot-in-a-wire heterostructures on Si(111)," Appl. Phys. Lett. 96(1), 013106 (2010), http:// dx.doi.org/10.1063/1.3284660.

13. W. Guo et al., "Catalyst-free InGaN/GaN nanowire light emitting diodes grown on (001) silicon by molecular beam epitaxy," Nano Lett. 10(9), 3355-3359 (2010), http://dx.doi .org/10.1021/nl101027x.

14. H. P. Nguyen et al., "Controlling electron overflow in phosphor-free $\mathrm{InGaN} / \mathrm{GaN}$ nanowire white light-emitting diodes," Nano Lett. 12(3), 1317-1323 (2012), http://dx.doi.org/10 $.1021 / \mathrm{nl} 203860 \mathrm{~b}$.

15. J.-H. Kang et al., "Defect-free GaAs/AlGaAs core-shell nanowires on Si substrates," Cryst. Growth Des. 11(7), 3109-3114 (2011), http://dx.doi.org/10.1021/cg2003657.

16. P. K. Mohseni et al., "GaP/GaAsP/GaP core-multishell nanowire heterostructures on (111) silicon," Nanotechnology 18(44), 445304 (2007), http://dx.doi.org/10.1088/0957-4484/ $18 / 44 / 445304$.

17. G. Koblmuller et al., "Self-induced growth of vertical free-standing InAs nanowires on $\mathrm{Si}$ (111) by molecular beam epitaxy," Nanotechnology 21(36), 365602 (2010), http://dx.doi .org/10.1088/0957-4484/21/36/365602.

18. M. Law et al., "Nanoribbon waveguides for subwavelength photonics integration," Science 305(5688), 1269-1273 (2004), http://dx.doi.org/10.1126/science.1100999.

19. L. K. van Vugt, S. Rühle, and D. Vanmaekelbergh, "Phase-correlated nondirectional laser emission from the end facets of a $\mathrm{ZnO}$ nanowire," Nano Lett. 6(12), 2707-2711 (2006), http://dx.doi.org/10.1021/nl0616227.

20. C. Klingshirn et al., "Excitonic ultraviolett lasing in $\mathrm{ZnO}$-based light emitting devices," Appl. Phys. Lett. 91(12), 126101 (2007), http://dx.doi.org/10.1063/1.2786585.

21. C. Klingshirn et al., "Room-temperature stimulated emission of $\mathrm{ZnO}$ : alternatives to excitonic lasing," Phys. Rev. B 75(11), 115203 (2007), http://dx.doi.org/10.1103/ PhysRevB.75.115203.

22. J. Fallert et al., "Random lasing in $\mathrm{ZnO}$ nanocrystals," J. Lumin. 129(12), 1685-1688 (2009), http://dx.doi.org/10.1016/j.jlumin.2009.02.022.

23. D. J. Gargas, M. E. Toimil-Molares, and P. Yang, "Imaging single $\mathrm{ZnO}$ vertical nanowire laser cavities using UV-laser scanning confocal microscopy," J. Am. Chem. Soc. 131(6), 2125-2127 (2009), http://dx.doi.org/10.1021/ja8092339. 
24. S. Chu et al., "Electrically pumped waveguide lasing from $\mathrm{ZnO}$ nanowires," Nat. Nanotechnol. 6(8), 506-510 (2011), http://dx.doi.org/10.1038/nnano.2011.97.

25. X. Duan et al., "Single-nanowire electrically driven lasers," Nature 421(6920), 241-245 (2003), http://dx.doi.org/10.1038/nature01353.

26. R. Agarwal, C. J. Barrelet, and C. M. Lieber, "Lasing in single cadmium sulfide nanowire optical cavities," Nano Lett. 5(5), 917-920 (2005), http://dx.doi.org/10.1021/n1050440u.

27. B. L. Cao et al., "Synthesis and lasing properties of highly ordered CdS nanowire arrays," Adv. Funct. Mater. 17(9), 1501-1506 (2007), http://dx.doi.org/10.1002/(ISSN)1616-3028.

28. B. Hua et al., "Single GaAs/GaAsP coaxial core-shell nanowire lasers," Nano Lett. 9(1), 112-116 (2009), http://dx.doi.org/10.1021/nl802636b.

29. A. H. Chin et al., "Near-infrared semiconductor subwavelength-wire lasers," Appl. Phys. Lett. 88(16), 163115 (2006), http://dx.doi.org/10.1063/1.2198017.

30. A. Pan et al., "Continuous alloy-composition spatial grading and superbroad wavelengthtunable nanowire lasers on a single chip," Nano Lett. 9(2), 784-788 (2009), http://dx.doi .org/10.1021/nl803456k.

31. R. Chen et al., "Nanolasers grown on silicon," Nat. Photon. 5(3), 170-175 (2011), http:// dx.doi.org/10.1038/nphoton.2010.315.

32. J. C. Johnson et al., "Single gallium nitride nanowire lasers," Nat. Mater. 1(2), 106-110 (2002), http://dx.doi.org/10.1038/nmat728.

33. H.-J. Choi et al., "Self-organized GaN quantum wire UV lasers," J. Phys. Chem. C 107(34), 8721-8725 (2003).

34. S. Gradečak et al., "GaN nanowire lasers with low lasing thresholds," Appl. Phys. Lett. 87(17), 173111 (2005), http://dx.doi.org/10.1063/1.2115087.

35. P. J. Pauzauskie, D. J. Sirbuly, and P. Yang, "Semiconductor nanowire ring resonator laser," Phys. Rev. Lett. 96(14), 143903 (2006), http://dx.doi.org/10.1103/PhysRevLett .96 .143903 .

36. M.-S. Hu et al., "Infrared lasing in InN nanobelts," Appl. Phys. Lett. 90(12), 123109 (2007), http://dx.doi.org/10.1063/1.2714291.

37. H.-G. Park et al., "Microstadium single-nanowire laser," Appl. Phys. Lett. 91(25), 251115 (2007), http://dx.doi.org/10.1063/1.2825470.

38. F. Qian et al., "Multi-quantum-well nanowire heterostructures for wavelength-controlled lasers," Nat. Mater. 7(9), 701-706 (2008), http://dx.doi.org/10.1038/nmat2253.

39. T. Kouno et al., "Two-dimensional light confinement in periodic InGaN/GaN nanocolumn arrays and optically pumped blue stimulated emission," Opt. Express 17(22), 2044020447 (2009), http://dx.doi.org/10.1364/OE.17.020440.

40. J. Heo, W. Guo, and P. Bhattacharya, "Monolithic single GaN nanowire laser with photonic crystal microcavity on silicon," Appl. Phys. Lett. 98(2), 021110 (2011), http://dx.doi .org/10.1063/1.3540688.

41. S. Ishizawa et al., "Optically pumped green $(530-560 \mathrm{~nm})$ stimulated emissions from InGaN/GaN multiple-quantum-well triangular-lattice nanocolumn arrays," Appl. Phys. Express 4(5), 055001 (2011), http://dx.doi.org/10.1143/APEX.4.055001.

42. A. Das et al., "Room temperature ultralow threshold GaN nanowire polariton laser," Phys. Rev. Lett. 107(6), 066405 (2011), http://dx.doi.org/10.1103/PhysRevLett.107 .066405 .

43. C. Y. Wu et al., "Plasmonic green nanolaser based on a metal-oxide-semiconductor structure," Nano Lett. 11(10), 4256-4260 (2011), http://dx.doi.org/10.1021/nl2022477.

44. S.-P. Chang et al., "Lasing action in gallium nitride quasicrystal nanorod arrays," Opt. Express 20(11), 12457-12462 (2012), http://dx.doi.org/10.1364/OE.20.012457.

45. Y. J. Lu et al., "Plasmonic nanolaser using epitaxially grown silver film," Science 337(6093), 450-453 (2012), http://dx.doi.org/10.1126/science.1223504.

46. Q. Li et al., "Single-mode GaN nanowire lasers," Opt. Express 20(16), 17873-17879 (2012), http://dx.doi.org/10.1364/OE.20.017873.

47. H. Xu et al., "Single-mode lasing of GaN nanowire-pairs," Appl. Phys. Lett. 101(11), 113106 (2012), http://dx.doi.org/10.1063/1.4751862.

48. H. Xu et al., "Gold substrate-induced single-mode lasing of GaN nanowires," Appl. Phys. Lett. 101(22), 221114 (2012), http://dx.doi.org/10.1063/1.4768300. 
49. T. Tawara et al., "Cavity polaritons in InGaN microcavities at room temperature," Phys. Rev. Lett. 92(25), 256402 (2004), http://dx.doi.org/10.1103/PhysRevLett.92.256402.

50. Y.-R. Wu, Y.-Y. Lin, and J. Singh, "InGaN light emitters: a comparison of quantum dot and quantum well based devices," in Conf. Lasers and Electro-Optics/Quantum Electronics and Laser Science Conference and Photonic Applications Systems Technologies, CLEO/QELS Poster Session III, JThA72, OSA Technical Digest (CD), San Jose, CA (2008).

51. W. Huang and F. Jain, "Enhanced optical gain in InGaN-AlGaN quantum wire and quantum dot lasers due to excitonic transitions," J. Appl. Phys. 87(10), 7354-7359 (2000), http://dx.doi.org/10.1063/1.372993.

52. N. Malkova and C. Ning, "Surface states of wurtzite semiconductor nanowires with identical lateral facets: a transfer-matrix approach," Phys. Rev. B 74(15), 155308 (2006), http://dx.doi.org/10.1103/PhysRevB.74.155308.

53. B. Beschoten et al., "Spin coherence and dephasing in GaN," Phys. Rev. B 63(12), 121202 (2001), http://dx.doi.org/10.1103/PhysRevB.63.121202.

54. G. Christmann et al., "Room temperature polariton lasing in a GaN/AlGaN multiple quantum well microcavity,” Appl. Phys. Lett. 93(5), 051102 (2008), http://dx.doi.org/10.1063/1 .2966369.

55. P. J. Pauzauskie and P. Yang, "Nanowire photonics," Mater. Today 9(10), 36-45 (2006), http://dx.doi.org/10.1016/S1369-7021(06)71652-2.

56. D. Saxena, S. Mokkapati, and C. Jagadish, "Semiconductor nanolasers," IEEE Photon. J. 4(2), 582-585 (2012).

57. R. Yan, D. Gargas, and P. Yang, "Nanowire photonics," Nat. Photon. 3(10), 569-576 (2009), http://dx.doi.org/10.1038/nphoton.2009.184.

58. D. Vanmaekelbergh and L. K. van Vugt, "ZnO nanowire lasers," Nanoscale 3(7), 2783 2800 (2011), http://dx.doi.org/10.1039/c1nr00013f.

59. M. A. Zimmler et al., "Optically pumped nanowire lasers: invited review," Semicond. Sci. Technol. 25(2), 024001 (2010), http://dx.doi.org/10.1088/0268-1242/25/2/024001.

60. Y. Ma and L. Tong, "Optically pumped semiconductor nanowire lasers," Front. Optoelectron. 5(3), 239-247 (2012), http://dx.doi.org/10.1007/s12200-012-0277-7.

61. C. Ning, "Semiconductor nanowire lasers," Chapter 12 in Advances in Semiconductor Lasers, J. J. Coleman, A. Catrina Bryce, and C. Jagadish, Eds., pp. 455-486, Academic Press, Burlington (2012).

62. R. Han-Youl, "Extraction efficiency in GaN nanorod light-emitting diodes investigated by finite-difference time-domain simulation," J. Korean Phys. Soc. 58(41), 878-882 (2011), http://dx.doi.org/10.3938/jkps.58.878.

63. Y.-J. Liu et al., "Improved performance of an InGaN-based light-emitting diode with a p-GaN/n-GaN barrier junction," IEEE J. Quantum Electron. 47(6), 755-761 (2011), http://dx.doi.org/10.1109/JQE.2011.2114330.

64. L.-B. Chang et al., "Effect of electron leakage on efficiency droop in wide-well InGaNbased light-emitting diodes," Appl. Phys. Express 4(1), 012106 (2011), http://dx.doi.org/ 10.1143/APEX.4.012106.

65. C.-H. Chiu et al., "Optical and electrical properties of GaN-based light emitting diodes grown on micro- and nano-scale patterned Si substrate," IEEE J. Quantum Electron. 47(7), 899-906 (2011), http://dx.doi.org/10.1109/JQE.2011.2114640.

66. H. P. T. Nguyen et al., "P-type modulation doped InGaN/GaN dot-in-a-wire white-lightemitting diodes monolithically grown on Si(111)," Nano Lett. 11(5), 1919-1924 (2011), http://dx.doi.org/10.1021/nl104536x.

67. S. J. Chang et al., "GaN Schottky barrier photodetectors," IEEE Sensors J. 10(10), 1609_ 1614 (2010), http://dx.doi.org/10.1109/JSEN.2010.2045889.

68. K. P. Korona et al., "Nitride-based photodetectors containing quantum wells in tunable electric fields," J. Optoelectron. Adv. Mater. 11(9), 1108-1114 (2009).

69. H. C. Lee et al., "AlInGaN metal-insulator-semiconductor photodetectors at UV-C 280 nm," Electrochem. Solid-State Lett. 12(10), H357-H360 (2009), http://dx.doi.org/ 10.1149/1.3182808. 
70. E. Muñoz, “(Al,In,Ga)N-based photodetectors. Some materials issues," Phys. Status Solidi B 244(8), 2859-2877 (2007), http://dx.doi.org/10.1002/(ISSN)1521-3951.

71. S. C. Jain et al., "III-nitrides: growth, characterization, and properties," J. Appl. Phys. 87(3), 965-1006 (2000), http://dx.doi.org/10.1063/1.371971.

72. M. A. Reshchikov and H. Morkoc, "Luminescence properties of defects in GaN," J. Appl. Phys. 97(6), 061301 (2005), http://dx.doi.org/10.1063/1.1868059.

73. S. Nakamura, "III-V nitride based light-emitting devices," Solid State Commun. 102(2-3), 237-248 (1997), http://dx.doi.org/10.1016/S0038-1098(96)00722-3.

74. L. Sugiura, "Dislocation motion in GaN light-emitting devices and its effect on device lifetime," J. Appl. Phys. 81(4), 1633-1638 (1997), http://dx.doi.org/10.1063/1.364018.

75. S. P. DenBaars, "Gallium-nitride-based materials for blue to ultraviolet optoelectronics devices," Proc. IEEE 85(11), 1740-1749 (1997), http://dx.doi.org/10.1109/5.649651.

76. T. Miyoshi et al., " $515 \mathrm{~nm}$ InGaN-based green laser diodes on c-Plane GaN substrate," Appl. Phys. Express 2(6), 062201 (2009), http://dx.doi.org/10.1143/APEX.2.062201.

77. A. Avramescu et al., "InGaN laser diodes with $50 \mathrm{~mW}$ output power emitting at $515 \mathrm{~nm}$," Appl. Phys. Lett. 95(7), 071103 (2009), http://dx.doi.org/10.1063/1.3206739.

78. A. Tyagi et al., "AlGaN-cladding free green semipolar $\mathrm{GaN}$ based laser diode with a lasing wavelength of 506.4 nm," Appl. Phys. Express 3(1), 011002 (2010), http://dx.doi.org/10 .1143/APEX.3.011002.

79. K. Okamoto, T. Tanaka, and M. Kubota, "High-efficiency continuous-wave operation of blue-green laser diodes based on nonpolar m-plane Gallium nitride," Appl. Phys. Express 1(7), 072201 (2008), http://dx.doi.org/10.1143/APEX.1.072201.

80. C.-Y. Huang et al., "Demonstration of $505 \mathrm{~nm}$ laser diodes using wavelength-stable semipolar (2021) InGaN/GaN quantum wells," Appl. Phys. Lett. 99(24), 241115 (2011), http:// dx.doi.org/10.1063/1.3666791.

81. F. Qian et al., "Gallium nitride-based nanowire radial heterostructures for nanophotonics," Nano Lett. 4(10), 1975-1979 (2004), http://dx.doi.org/10.1021/n10487774.

82. G. T. Wang et al., "III-nitride nanowires: novel materials for solid-state lighting," Proc. SPIE 7954, 79540T (2011), http://dx.doi.org/10.1117/12.872776.

83. G. T. Wang et al., "III-Nitride nanowires: novel materials for lighting and photovoltaics," in Photonics North Conf. (GREEN-7-41-1), Montreal, Quebec (2012).

84. W. Guo et al., "Auger recombination in III-Nitride nanowires and its effect on nanowire light-emitting diode characteristics," Nano Lett. 11(4), 1434-1438 (2011), http://dx.doi .org/10.1021/nl103649d.

85. M.-H. Kim et al., "Origin of efficiency droop in GaN-based light-emitting diodes," Appl. Phys. Lett. 91(18), 183507 (2007), http://dx.doi.org/10.1063/1.2800290.

86. X. Ni et al., "The effect of ballistic and quasi-ballistic electrons on the efficiency droop of InGaN light emitting diodes," Phys. Status Solidi RRL 4(8-9), 194-196 (2010).

87. J. Xie et al., "On the efficiency droop in InGaN multiple quantum well blue light emitting diodes and its reduction with p-doped quantum well barriers," Appl. Phys. Lett. 93(12), 121107 (2008), http://dx.doi.org/10.1063/1.2988324.

88. Y. C. Shen et al., "Auger recombination in InGaN measured by photoluminescence," Appl. Phys. Lett. 91(14), 141101 (2007), http://dx.doi.org/10.1063/1.2785135.

89. M. Zhang et al., "Direct measurement of auger recombination in $\mathrm{In} 0.1 \mathrm{Ga} 0.9 \mathrm{~N} / \mathrm{GaN}$ quantum wells and its impact on the efficiency of $\mathrm{In} 0.1 \mathrm{Ga} 0.9 \mathrm{~N} / \mathrm{GaN}$ multiple quantum well light emitting diodes," Appl. Phys. Lett. 95(20), 201108 (2009), http://dx.doi.org/10.1063/ 1.3266520 .

90. S. Chevtchenko et al., "Surface band bending of a-plane GaN studied by scanning Kelvin probe microscopy," Appl. Phys. Lett. 88(12), 122104 (2006), http://dx.doi.org/10.1063/1 .2188589 .

91. N. A. Sanford et al., "Steady-state and transient photoconductivity in c-axis GaN nanowires grown by nitrogen-plasma-assisted molecular beam epitaxy," J. Appl. Phys. 107(3), 034318 (2010), http://dx.doi.org/10.1063/1.3275888.

92. C. Guthy, C.-Y. Nam, and J. E. Fischer, "Unusually low thermal conductivity of gallium nitride nanowires," J. Appl. Phys. 103(6), 064319 (2008), http://dx.doi.org/10.1063/1 .2894907 . 
93. J. Zou, "Lattice thermal conductivity of freestanding gallium nitride nanowires," J. Appl. Phys. 108(3), 034324 (2010), http://dx.doi.org/10.1063/1.3463358.

94. Z. Wang et al., "Atomistic simulation of the size and orientation dependences of thermal conductivity in GaN nanowires," Appl. Phys. Lett. 90(16), 161923 (2007), http://dx.doi .org/10.1063/1.2730747.

95. M. L. Colussi, R. J. Baierle, and R. H. Miwa, "Doping effects of C, Si and Ge in wurtzite [0001] GaN, AlN, and InN nanowires," J. Appl. Phys. 110(3), 033709 (2011), http://dx.doi .org/10.1063/1.3607280.

96. S. Zhao et al., "Tuning the surface charge properties of epitaxial InN nanowires," Nano Lett. 12(6), 2877-2882 (2012), http://dx.doi.org/10.1021/nl300476d.

97. E. M. Purcell, "Spontaneous emission probabilities at radio frequencies," Phys. Rev. 69(11-12), 681 (1946).

98. C. Z. Ning, "Nanolasers based on nanowires and surface plasmons," SPIE Newsroom (2009), http://dx.doi.org/10.1117/2.1200901.1486.

99. Y.-Z. Huang, Z. Pan, and R.-H. Wu, "Analysis of the optical confinement factor in semiconductor lasers," J. Appl. Phys. 79(8), 3827-3830 (1996), http://dx.doi.org/10.1063/1 .361809 .

100. Y. Z. Huang, "Comparison of modal gain and material gain for strong guiding slab waveguides," Proc. IEE Optoelectron. 148(3), 131-133 (2001).

101. T. D. Visser et al., "Confinement factors and gain in optical amplifiers," IEEE J. Quantum Electron. 33(10), 1763-1766 (1997), http://dx.doi.org/10.1109/3.631280.

102. A. V. Maslov and C. Z. Ning, "Modal gain in a semiconductor nanowire laser with anisotropic bandstructure," IEEE J. Quantum Electron. 40(10), 1389-1397 (2004), http://dx.doi .org/10.1109/JQE.2004.834767.

103. J. T. Robinson et al., "First-principle derivation of gain in high-index-contrast waveguides," Opt. Express 16(21), 16659-16669 (2008), http://dx.doi.org/10.1364/OE.16 .016659 .

104. L. A. Coldren and S. W. Corzine, Diode Lasers and Photonic Integrated Circuits, p. 446, John Wiley \& Sons, New York (1995).

105. A. V. Maslov and C. Z. Ning, "GaN nanowire lasers," Chapter 21 in Nitride Semiconductor Devices: Principles and Simulation, J. Piprek, Ed., pp. 467-491, Wiley-VCH Verlag GmbH \& Co. KGaA, Weinheim (2007).

106. C. Z. Ning, "Semiconductor nanolasers," Phys. Status Solidi B 247(4), 774-788 (2010).

107. A. V. Maslov and C. Z. Ning, "Reflection of guided modes in a semiconductor nanowire laser," Appl. Phys. Lett. 83(6), 1237-1239 (2003), http://dx.doi.org/10.1063/1 .1599037.

108. A. W. Snyder and J. D. Love, Optical Waveguide Theory, Chapman and Hall, London (1983).

109. R. R. Burton et al., "Modelling of diffraction in pillar vertical cavity surface-emitting lasers with embedded Bragg layers," Opt. Quantum Electron. 28(11), 1677-1684 (1996), http:// dx.doi.org/10.1007/BF00331057.

110. A.-L. Henneghien et al., "Simulation of waveguiding and emitting properties of semiconductor nanowires with hexagonal or circular sections," J. Opt. Soc. Am. B 26(12), 2396-2403 (2009), http://dx.doi.org/10.1364/JOSAB.26.002396.

111. S.-W. Chang and S. L. Chuang, "Fundamental formulation for plasmonic nanolasers," IEEE J. Quantum Electron. 45(8), 1014-1023 (2009), http://dx.doi.org/10.1109/JQE .2009 .2017210 .

112. S.-W. Chang, T.-R. Lin, and S. L. Chuang, "Theory of plasmonic Fabry-Perot nanolasers," Opt. Express 18(14), 15039-15053 (2010), http://dx.doi.org/10.1364/OE.18.015039.

113. S.-W. Chang and S. L. Chuang, "Normal modes for plasmonic nanolasers with dispersive and inhomogeneous media," Opt. Lett. 34(1), 91-93 (2009), http://dx.doi.org/10.1364/OL .34.000091.

114. T. W. George et al., "Highly aligned, template-free growth and characterization of vertical GaN nanowires on sapphire by metal-organic chemical vapour deposition," Nanotechnology 17(23), 5773-5780 (2006), http://dx.doi.org/10.1088/0957-4484/17/23/ 011. 
115. T. Kuykendall et al., "Crystallographic alignment of high-density gallium nitride nanowire arrays," Nat. Mater. 3(8), 524-528 (2004), http://dx.doi.org/10.1038/nmat1177.

116. T. Kuykendall et al., "Complete composition tunability of InGaN nanowires using a combinatorial approach," Nat. Mater. 6(12), 951-956 (2007), http://dx.doi.org/10.1038/ nmat2037.

117. R. Armitage and K. Tsubaki, "Multicolour luminescence from InGaN quantum wells grown over GaN nanowire arrays by molecular-beam epitaxy," Nanotechnology 21(19), 195202 (2010), http://dx.doi.org/10.1088/0957-4484/21/19/195202.

118. Y. Kawakami et al., "Origin of high oscillator strength in green-emitting InGaN/GaN nanocolumns," Appl. Phys. Lett. 89(16), 163124 (2006), http://dx.doi.org/10.1063/1 .2363958 .

119. H.-M. Kim et al., "Formation of InGaN nanorods with indium mole fractions by hydride vapor phase epitaxy," Phys. Status Solidi B 241(12), 2802-2805 (2004), http://dx.doi.org/ $10.1002 /($ ISSN)1521-3951.

120. H.-M. Kim et al., "InGaN nanorods grown on (111) silicon substrate by hydride vapor phase epitaxy," Chem. Phys. Lett. 380(1-2), 181-184 (2003), http://dx.doi.org/10 .1016/j.cplett.2003.09.020.

121. F. Qian et al., "Core/multishell nanowire heterostructures as multicolor, high-efficiency light-emitting diodes," Nano Lett. 5(11), 2287-2291 (2005), http://dx.doi.org/10.1021/ nl051689e.

122. A. Kikuchi et al., "InGaN/GaN multiple quantum disk nanocolumn light-emitting diodes grown on (111) Si substrate," Jpn. J. Appl. Phys. 43(12A), L1524-L1526 (2004), http://dx .doi.org/10.1143/JJAP.43.L1524.

123. H.-W. Lin et al., "InGaN/GaN nanorod array white light-emitting diode," Appl. Phys. Lett. 97(7), 073101-073103 (2010), http://dx.doi.org/10.1063/1.3478515.

124. M. Zervos et al., "Low temperature growth of $\operatorname{In}_{2} \mathrm{O}_{3}$ and $\mathrm{InN}$ nanocrystals on $\mathrm{Si}(111)$ via chemical vapour deposition based on the sublimation of $\mathrm{NH}_{4} \mathrm{Cl}$ in In," Nanoscale Res. Lett. 4(6), 491-497 (2009), http://dx.doi.org/10.1007/s11671-009-9266-1.

125. J. Grandal et al., "Accommodation mechanism of InN nanocolumns grown on $\mathrm{Si}(111)$ substrates by molecular beam epitaxy," Appl. Phys. Lett. 91(2), 021902 (2007), http:// dx.doi.org/10.1063/1.2756293.

126. I. Shalish et al., "Epitaxial catalyst-free growth of InN nanorods on c-plane sapphire," Nanoscale Res. Lett. 4(6), 532-537 (2009), http://dx.doi.org/10.1007/s11671-009-9276-z.

127. K. R. Wang et al., "InN nanotips as excellent field emitters," Appl. Phys. Lett. 92(12), 123105 (2008), http://dx.doi.org/10.1063/1.2897305.

128. R. F. Oulton et al., "A hybrid plasmonic waveguide for subwavelength confinement and long-range propagation," Nat. Photonics 2(8), 496-500 (2008), http://dx.doi.org/10.1038/ nphoton.2008.131.

129. R. F. Oulton et al., "Plasmon lasers at deep subwavelength scale," Nature 461(7264), 629-632 (2009), http://dx.doi.org/10.1038/nature08364.

130. L. Chen and E. Towe, "Nanowire lasers with distributed-Bragg-reflector mirrors," Appl. Phys. Lett. 89(5), 053125 (2006), http://dx.doi.org/10.1063/1.2245219.

131. L. Tong, J. Lou, and E. Mazur, "Single-mode guiding properties of subwavelengthdiameter silica and silicon wire waveguides," Opt. Express 12(6), 1025-1035 (2004), http://dx.doi.org/10.1364/OPEX.12.001025.

132. S.-S. Wang et al., "Modeling endface output patterns of optical micro/nanofibers," Opt. Express 16(12), 8887-8895 (2008), http://dx.doi.org/10.1364/OE.16.008887.

133. A. E. Siegman, "Laser beams and resonators: beyond the 1960s," IEEE J. Sel. Top. Quantum Electron. 6(6), 1389-1399 (2000), http://dx.doi.org/10.1109/2944.902193.

134. H. Cao, "Review on latest developments in random lasers with coherent feedback," J. Phys. A Math. Gen. 38(49), 10497-10535 (2005), http://dx.doi.org/10.1088/0305-4470/38/49/ 004.

135. A. V. Kavokin et al., "Vertical cavity surface emitting terahertz laser," Phys. Rev. Lett. 108(19), 197401 (2012), http://dx.doi.org/10.1103/PhysRevLett.108.197401.

136. H. Kogelnik and C. V. Shank, "Coupled-wave theory of distributed feedback lasers," J. Appl. Phys. 43(5), 2327-2335 (1972), http://dx.doi.org/10.1063/1.1661499. 
137. M. Imada et al., "Coherent two-dimensional lasing action in surface-emitting laser with triangular-lattice photonic crystal structure," Appl. Phys. Lett. 75(3), 316-318 (1999), http://dx.doi.org/10.1063/1.124361.

138. H. Matsubara et al., "GaN photonic-crystal surface-emitting laser at blue-violet wavelengths," Science 319(5862), 445-447 (2008), http://dx.doi.org/10.1126/science.1150413.

139. K. Kishino and S. Arai, "Integrated lasers," Chapter 11 in Handbook of Semiconductor Lasers and Photonic Integrated Circuits, Y. Suematsu and A. R. Adams, Eds., p. 350, Chapman \& Hall, London (1994).

140. Y. Yamamoto, S. Machida, and G. Björk, "Microcavity semiconductor laser with enhanced spontaneous emission," Phys. Rev. A 44(1), 657-668 (1991), http://dx.doi.org/10.1103/ PhysRevA.44.657.

141. S. Strauf et al., "Self-tuned quantum dot gain in photonic crystal lasers," Phys. Rev. Lett. 96(12), 127404 (2006), http://dx.doi.org/10.1103/PhysRevLett.96.127404.

142. H. Altug, D. Englund, and J. Vuckovic, "Ultrafast photonic crystal nanocavity laser," Nat. Phys. 2(7), 484-488 (2006), http://dx.doi.org/10.1038/nphys343.

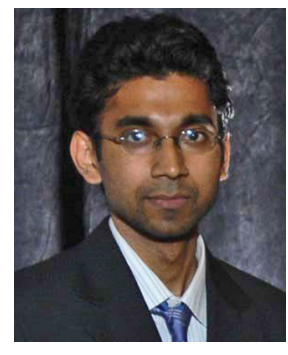

Shamsul Arafin received his B.Sc. degree in electrical and electronics engineering from Bangladesh University of Engineering and Technology (BUET), Bangladesh in 2005 and M.Sc. degree in communication technology from Universität Ulm, Germany in 2008. He received his $\mathrm{PhD}$ degree from Technische Universität München, Walter Schottky Institut Germany, in early 2012. Then, he joined the nanophotonics group of Electrical and Computer Engineering Department at McGill University as a post-doc fellow. His current research interests include semiconductor lasers, nanophotonic devices, and integrated photonics.

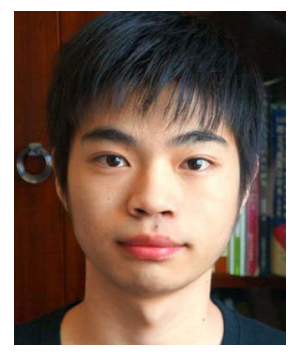

Xianhe Liu obtained his B.Sc. degree from Huaqiao University in 2012. Now, he is a $\mathrm{PhD}$ student in the nanophotonics group led by Professor Zetian Mi at McGill University. His research interests cover III-nitride based nanolasers and ultraviolet light-emitting diodes.

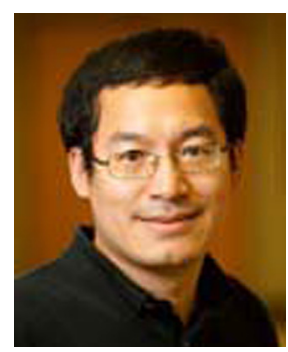

Zetian Mi is an associate professor in the Department of Electrical and Computer Engineering at McGill University. $\mathrm{He}$ is also an associate member in the Department of Physics at McGill. He received his PhD degree in applied physics from the University of Michigan in 2006. His teaching and research interests are in the areas of III-nitride semiconductors, solar fuels, nanophotonics, and nanoelectronics. He has published seven book chapters and more than 200 refereed journal and conference papers. He has received the Hydro-Quebec Nano-Engineering Scholar Award in 2009, the William Dawson Scholar Award in 2011, and the Christophe Pierre Award for Research Excellence (Early Career) in 2012 at McGill University. He has also received the Young Investigator Award from the 27th North American Molecular Beam Epitaxy Conference. He currently serves as the associate editor of IEEE Journal of Lightwave Technology. 